\title{
TOWARDS A UNIVERSALITY PICTURE FOR THE RELAXATION TO EQUILIBRIUM OF KINETICALLY CONSTRAINED MODELS ${ }^{1}$
}

\author{
By FABIO MARTINELli AND CRISTINA TONINELli
}

\author{
Università Roma Tre and CNRS-Universités Paris VI-VII
}

Recent years have seen a great deal of progress in our understanding of bootstrap percolation models, a particular class of monotone cellular automata. In the two-dimensional lattice $\mathbb{Z}^{2}$, there is now a quite satisfactory understanding of their evolution starting from a random initial condition, with a strikingly beautiful universality picture for their critical behavior. Much less is known for their nonmonotone stochastic counterpart, namely kinetically constrained models (KCM). In KCM, each vertex is resampled (independently) at rate one by tossing a $p$-coin iff it can be infected in the next step by the bootstrap model. In particular, an infection can also heal, hence the nonmonotonicity. Besides the connection with bootstrap percolation, $\mathrm{KCM}$ have an interest in their own as they feature some of the most striking features of the liquid/glass transition, a major and still largely open problem in condensed matter physics. In this paper, we pave the way towards proving universality results for the characteristic time scales of KCM. Our novel and general approach gives the right tools to establish a close connection between the critical scaling of characteristic time scales for KCM and the scaling of the critical length in critical bootstrap models. When applied to the FredricksonAndersen $k$-facilitated models in dimension $d \geq 2$, among the most studied $\mathrm{KCM}$, and to the Gravner-Griffeath model, our results are close to optimal.

1. Introduction. In recent years, remarkable progress has been obtained in understanding the behaviour of a particular class of monotone cellular automata known as bootstrap percolation. A general bootstrap cellular automaton [7] is specified by its update family $\mathcal{U}=\left\{U_{1}, \ldots, U_{m}\right\}$ of finite subsets of $\mathbb{Z}^{d} \backslash 0$. Once $\mathcal{U}$ is given, the $\mathcal{U}$-bootstrap percolation process is as follows. Given a set $A \subset \mathbb{Z}^{d}$ of initially infected vertices, set $A_{0}=A$, and define recursively for each $t \in \mathbb{N}$,

$$
A_{t+1}=A_{t} \cup\left\{x \in \mathbb{Z}^{d}: x+U_{k} \subset A_{t} \text { for some } k \in(1, \ldots m)\right\} .
$$

In other words a vertex $x$ becomes infected at time $t+1$ if the translate by $x$ of at least one element of the update family is already entirely infected at time $t$, and infected vertices remain infected forever. We write $[A]_{\mathcal{U}}:=\bigcup_{t \geq 0} A_{t}$ for the closure of $A$ under the $\mathcal{U}$-bootstrap process.

Received January 2017; revised December 2017.

${ }^{1}$ Supported by the ERC Starting Grant 680275 MALIG, by the ANR-15-CE40-0020-01 grant LSD and by the PRIN 20155PAWZB "Large Scale Random Structures."

MSC2010 subject classifications. Primary 60K35; secondary 60J27.

Key words and phrases. Glauber dynamics, kinetically constrained models, spectral gap, bootstrap percolation. 
A much studied example is the classical $r$-neighbour model (see [3] and references therein) in which a vertex gets infected if at least $r$ among its nearest neighbours are infected, namely the update family is formed by all the $r$-subsets of the set of the nearest neighbours of the origin.

A central problem for bootstrap models is their long-time evolution when the initial infected set $A_{0}$ is $\mathbb{P}_{q}(\cdot)$-random, that is, each vertex of $\mathbb{Z}^{d}$, independently from the other vertices, is initially declared to be infected with probability $q \in$ $(0,1)$. A key quantity is then the critical percolation threshold

$$
q_{c}(\mathcal{U}):=\inf \left\{q: \mathbb{P}_{q}\left([A]=\mathbb{Z}^{d}\right)=1\right\}
$$

Two closely related quantities are $T_{c}(q ; \mathcal{U})$ and $L_{c}(q ; \mathcal{U})$ defined as follows.

Definition 1.1. Let $\tau_{\mathrm{BP}}=\min \left\{t: 0 \in[A]_{t}\right\}$ be the infection time of the origin. Then

$$
T_{c}(q ; \mathcal{U})=\inf \left\{t \geq 0: \mathbb{P}_{q}\left(\tau_{\mathrm{BP}} \geq t\right) \leq 1 / 2\right\}
$$

To define $L_{c}(q ; \mathcal{U})$, let us consider the bootstrap percolation process on the $d$ dimensional torus $\mathbb{Z}_{n}^{d} \subset \mathbb{Z}^{d}$ of linear size $n$, and let $q_{c}(n ; \mathcal{U})$ be the smallest $q$ such that with probability at least $1 / 2$ the whole torus is eventually infected. Then

$$
L_{c}(q, \mathcal{U}):=\min \left\{n: q_{c}(n, \mathcal{U}) \leq q\right\}
$$

In $[2,5,7]$ beautiful universality results for general $\mathcal{U}$-bootstrap percolation processes in two dimensions satisfying $q_{c}(\mathcal{U})=0$ have been established, yielding in particular the sharp scaling behaviour of $T_{c}(q ; \mathcal{U}), L_{c}(q ; \mathcal{U})$ as $q \rightarrow 0$. For a nice review of these results, we refer the reader to [26], Section 1. It follows in particular [5], Theorems 1.4, 1.5, that in two dimensions

$$
0<\liminf _{q \rightarrow 0} \frac{\log \left(T_{c}(q ; \mathcal{U})\right)}{\log \left(L_{c}(q ; \mathcal{U})\right)} \leq \limsup _{q \rightarrow 0} \frac{\log \left(T_{c}(q ; \mathcal{U})\right)}{\log \left(L_{c}(q ; \mathcal{U})\right)}<+\infty
$$

and in this sense one can say that $T_{c}(q ; \mathcal{U})$ and $L_{c}(q ; \mathcal{U})$ have the same scaling behavior.

A quite natural stochastic counterpart of bootstrap percolation models are particular interacting particle systems known as kinetically constrained models (KCM). Given a $\mathcal{U}$-bootstrap model, the associated $\mathrm{KCM}$ is the continuous-time reversible Markov process on $\Omega=\{0,1\}^{\mathbb{Z}^{d}}$ constructed as follows. Denote by $\omega \in \Omega$ the current configuration of the process and call a vertex $x$ infected if $\omega_{x}=0$. Then each vertex $x$, with rate one and independently across $\mathbb{Z}^{d}$, is resampled by tossing a $p$-coin $[\operatorname{Prob}(1)=p]$ iff the translate by $x$ of at least one element of the update family $\mathcal{U}$ is already entirely infected for $\omega$. In other words, the state $\omega_{x}$ of the vertex $x$ is allowed to be resampled iff it was infectable in $\omega$ by the bootstrap process [8]. 
It is easy to check that such a process is reversible w.r.t. the Bernoulli $(p)$ product measure $\mu$ on $\Omega$. Notice that if $q:=1-p \ll 1$, it is very unlikely for a vertex to become infected (even if it would have been infected by the bootstrap process). Observe moreover that infected vertices may heal. The latter feature implies, in particular, that the $\mathrm{KCM}$ is not monotone/attractive, a fact that rules out several powerful tools from interacting particle systems theory like monotone coupling and censoring.

Besides the connection with cellular automata, KCM are of interest in their own. They have been in fact introduced in the physics literature in the 1980s to model the liquid/glass transition, a major and still largely open problem in condensed matter physics [4]. Extensive numerical simulations indicate that KCM display a remarkable glassy behavior, including heterogeneous dynamics, the occurrence of ergodicity breaking transitions, multiple invariant measures and anomalously long-time scales (see, e.g., [18] and references therein).

It has been proved in [8] that the KCM undergoes an ergodicity breaking transition at $q_{c}(\mathcal{U})$ and a major problem, both from the physical and mathematical point of view, is to determine the precise divergence of its characteristic time scales when $q \downarrow q_{c}$. A natural time scale is the mean hitting time $\mathbb{E}_{\mu}\left(\tau_{0}\right)$, where $\mathbb{E}_{\mu}(\cdot)$ is the average w.r.t. to the law of the stationary KCM process with initial law $\mu$ and

$$
\tau_{0}=\inf \left\{t \geq 0: \omega_{0}(t)=0\right\}
$$

For all those $\mathrm{KCM}$ whose update family $\mathcal{U}$ satisfies $q_{c}(\mathcal{U})=0$, one can then ask whether the scaling of $\mathbb{E}_{\mu}\left(\tau_{0}\right)$ as $q \downarrow 0$ is related to that of $T_{c}(q ; \mathcal{U}), L_{c}(q ; \mathcal{U})$. It is possible to prove (see Lemma 4.3) that there exists $\delta=\delta(\mathcal{U}) \in(0,1)$ such that, for all $q$ small enough,

$$
\mathbb{E}_{\mu}\left(\tau_{0}\right) \geq \delta \mathbb{E}_{q}\left(\tau_{\mathrm{BP}}\right) \geq \frac{\delta}{2} T_{c}(q ; \mathcal{U})
$$

So far, the best general upper bound on $\mathbb{E}_{\mu}\left(\tau_{0}\right)$ is a much poorer one of the form [8]

$$
\mathbb{E}_{\mu}\left(\tau_{0}\right) \leq e^{O\left(L_{c}(q ; \mathcal{U})^{d}\right)}
$$

Although this bound has been greatly improved for special choices of the update family $\mathcal{U}$, yielding in some cases the correct behavior (cf. [10, 13, 14]), for general $\mathrm{KCM}$ and contrary to the situation of bootstrap percolation in two dimensions, there is yet no universality picture for the scaling of $\mathbb{E}_{\mu}\left(\tau_{0}\right)$.

The present paper represents the first step of a general project concerning KCM with update family $\mathcal{U}$ satisfying $q_{c}(\mathcal{U})=0$, with the aim of establishing universality results on the scaling of $\mathbb{E}_{\mu}\left(\tau_{0}\right)$ as $q \rightarrow 0$ analogous to those proved within bootstrap percolation.

At the beginning of this program, in [26], Section 2, some conjectures were put forward, jointly with us, on the scaling of $\mathbb{E}_{\mu}\left(\tau_{0}\right)$. In particular, it was suggested 
that for $\mathrm{KCM}$ it is necessary to introduce a more refined classification of the universality classes in order to take into account the effect of the possible presence of energy barriers in the dynamics. By energy barriers, we mean very unlikely states with an anomalous amount of infection which are typically visited by the stationary KCM process before infecting the origin. More specifically, it was argued that energy barriers could induce a very different scaling of $\mathbb{E}_{\mu}\left(\tau_{0}\right)$ w.r.t. to that of $T_{c}(q ; \mathcal{U})$ for all those models for which the characteristic bootstrap percolation critical droplets are constrained to move inside a cone. Examples include the two-dimensional East [13] with $\mathcal{U}$ consisting of the 1 -subsets of $\bigcup_{i=1}^{d}\left\{-\vec{e}_{i}\right\}$ and Duarte-KCM model $[6,15,27]$ where $\mathcal{U}=2$-subsets of $\left\{\overrightarrow{-} e_{1}, \pm \vec{e}_{2}\right\}$. Significant progresses in this direction have been obtained after this work has been completed [23].

The main purpose of this paper is twofold. First, we envisage a general and novel approach to prove Poincaré inequalities for KCM, with the ultimate goal of finding the exact scaling of $\mathbb{E}_{\mu}\left(\tau_{0}\right)$ for a very large class of update families $\mathcal{U}$ with $q_{c}(\mathcal{U})=0$. For example, building upon the strategy and techniques developed in Sections 2 and 3, the following result has been recently established.

THEOREM 1 ([24]). For the so-called critical $\alpha$-unrooted KCM in two dimensions (see [26] for the appropriate definition),

$$
\mathbb{E}_{\mu}\left(\tau_{0}\right)=O\left(L_{c}(q ; \mathcal{U})^{\beta(q)}\right), \quad \beta(q)=\operatorname{poly}\left(\log \log L_{c}(q ; \mathcal{U})\right) \text { as } q \rightarrow 0
$$

Second, we want to greatly improve the existing upper bounds on $\mathbb{E}_{\mu}\left(\tau_{0}\right)$ for the most studied KCM on $\mathbb{Z}^{d}$, in any dimension $d \geq 2$, namely the FredricksonAndersen $k$-facilitated model (FA-kf) [1]. Its update family consists of the $k$-sets of the neighbors of the origin and, therefore, its associated bootstrap percolation version is the well-known $k$-neighbour model. We also test the flexibility of our techniques by briefly analysing the kinetically constrained version of the wellknown Gravner-Griffeath bootstrap percolation model on $\mathbb{Z}^{2}[19,20]$. In this case, $\mathcal{U}$ consists of the 3 -subsets of the set formed by the neighbours of the origin together with the vertices $( \pm 2,0)$ and it is known that the bootstrap process features a striking anisotropy. In both cases, our main result (see Theorem 4.4) establishes a tight connection between $\mathbb{E}_{\mu}\left(\tau_{0}\right)$ and $L_{c}(q ; \mathcal{U})$.

1.1. Main results and plan of the paper. In Section 2, after introducing the relevant notation and motivated by the connection between $\mathbb{E}_{\mu}\left(\tau_{0}\right)$ and the Poincaré inequality, we prove our first main result (Theorem 2). It establishes a (constrained) Poincaré inequality for very general KCM satisfying a rather flexible condition involving the range of the update family $\mathcal{U}$ and the probability that an update is feasible. Constrained Poincaré inequalities for KCM, implying a positive spectral gap and exponential mixing, have already been established [8], mainly using the so-called halving method. Here, inspired by our previous analysis of KCM on trees 
$[10,25]$, we develop an alternative method which, besides being more natural and direct, applies as well to update families with a large (depending on $q$ ) or infinite number of elements. As an example, in Section 2.5 we prove a Poincaré inequality for the $\mathrm{KCM}$ for which the constraint requires that the oriented neighbours of the to-be-updated vertex belong to an infinite cluster of infected vertices.

Section 3, and its main outcomes summarised in Corollary 3.9, is somehow the core of the work. By applying Theorem 2 together with a renormalisation argument and canonical-paths arguments, we prove a sharp bound on the best constant in the Poincaré inequality for general KCM. This bound involves the probability of occurrence of a critical droplet (in the bootstrap percolation language) together with certain congestion constants related to the cost of moving around the droplet. In this section, we made an effort to keep the framework as general as possible, in order to construct a very flexible tool that can be applied to any choice of constraints in any dimension.

In Section 4.1, we introduce the Fredrickson-Andersen $k$-facilitated (FA-kf) and the Gravner-Griffeath kinetically constrained (GG-KCM) models and state our main result Theorem 4.4 for the scaling of $\mathbb{E}\left(\tau_{0}\left(\mathbb{Z}^{d} ; \mathcal{U}\right)\right)$ in these cases. Finally, in Section 5 we prove Theorem 4.4 by bounding (model by model) the congestion constants appearing in the key inequality of Corollary 3.9.

\section{A constrained Poincaré inequality for product measures.}

2.1. Notation. For any integer $n$, we will write $[n]$ for the set $\{1,2, \ldots, n\}$. Given $x=\left(x_{1}, \ldots, x_{d}\right) \in \mathbb{Z}^{d}$ we denote its $\ell^{1}$-norm by $\|x\|_{1}=\sum_{i=1}^{d}\left|x_{i}\right|$ and by $d_{1}(\cdot, \cdot)$ the associated distance function. Given two vertices $x \neq y$, we will say that $x$ precedes $y$ and we will write $x \prec y$ if $x_{i} \leq y_{i}$ for all $i \in[d]$. The collection $\mathcal{B}=\left\{\vec{e}_{1}, \vec{e}_{2}, \ldots, \vec{e}_{d}\right\}$ will denote the canonical basis of $\mathbb{Z}^{d}$. Given a set $\Lambda \subset \mathbb{Z}^{d}$, we define its external boundary as the set

$$
\partial \Lambda=\left\{y \in \mathbb{Z}^{d} \backslash \Lambda: \exists x \in \Lambda \text { with }\|x-y\|_{1}=1\right\} .
$$

2.2. The probability space. Given a finite set $S$ and $\Lambda \subseteq \mathbb{Z}^{d}$, we will denote by $\Omega_{\Lambda}$ the product space $S^{\Lambda}$ endowed with the product topology. Given $V \subset \Lambda$ and $\omega \in \Omega_{\Lambda}$ we will write $\omega_{V}$ for the restriction of $\omega$ to $V$. Finally, we will denote by $\mu_{\Lambda}$ the product measure $\mu_{\Lambda}=\bigotimes_{x \in \Lambda} \hat{\mu}_{x}$ on $\Omega_{\Lambda}$ where, $\forall x \in \mathbb{Z}^{d}$, we set $\hat{\mu}_{x}=$ $\hat{\mu}$ with $\hat{\mu}$ a probability measure on $S$ which w.l.o.g. we assume to be positive. Expectation and variance w.r.t. $\mu_{\Lambda}$ are denoted by $\mathbb{E}_{\Lambda}(\cdot), \operatorname{Var}_{\Lambda}(\cdot)$, respectively. If $\Lambda=\mathbb{Z}^{d}$, the subscript $\Lambda$ will be dropped from the notation.

In several applications, the probability space $(S, \hat{\mu})$ will be the "particle space" $S=\{0,1\}^{V}$ where $V$ is a finite subset (a "block" as it is sometimes called) of $\mathbb{Z}^{d}$ and $\hat{\mu}=\bigotimes_{x \in V} B(p), B(p)$ being the $p$-Bernoulli measure. 
2.3. The constraints. For each $x \in \mathbb{Z}^{d}$, let $\Delta_{x} \subset \mathbb{Z}^{d} \backslash\{x\}$ be a finite set, let $\mathcal{A}_{x}$ be an event depending on the variables $\left\{\omega_{y}\right\}_{y \in \Delta_{x}}$ and let $c_{x}$ be its indicator function. By construction, $c_{x}$ does not depend on $\omega_{x}$. In the sequel, we will refer to $c_{x}$ as the constraint at $x$ and to $\varepsilon_{x}:=\mu\left(1-c_{x}\right)=\mu\left(\mathcal{A}_{x}^{c}\right)$ and $\Delta_{x}$ as its failure probability and support respectively. In our approach based on a martingale decomposition of the variance $\operatorname{Var}(f)$ of any local function $f: \Omega \mapsto \mathbb{R}$, a key role is played by constraints satisfying the following exterior condition.

DEFINITION 2.1 (Exterior condition). Given an exhausting collection of subsets $\left\{V_{n}\right\}_{n \in \mathbb{Z}}$ of $\mathbb{Z}^{d}$ (i.e., $V_{n} \subset V_{n+1}$ for all $n$ and $\bigcup_{n} V_{n}=\mathbb{Z}^{d}$ ), let $\mathcal{L}_{n}:=V_{n} \backslash V_{n-1}$ be the $n$ th-shell and, for any $x \in \mathcal{L}_{n}$, let the exterior of $x$ be the set Ext $_{x}:=$ $\bigcup_{j=n+1}^{\infty} \mathcal{L}_{j}$. We then say that the family of constraints $\left\{c_{x}\right\}_{x \in \mathbb{Z}^{d}}$ satisfies the exterior condition w.r.t. $\left\{V_{n}\right\}_{n=-\infty}^{\infty}$ if $\Delta_{x} \subset \operatorname{Ext}_{x}$ for all $x$. We will say that $\left\{c_{x}\right\}_{x \in \mathbb{Z}^{d}}$ satisfies the exterior condition if there exists a family of sets $\left\{V_{n}\right\}_{n \in \mathbb{Z}}$ as above such that $\left\{c_{x}\right\}_{x \in \mathbb{Z}^{d}}$ satisfies the exterior condition w.r.t. $\left\{V_{n}\right\}_{n \in \mathbb{Z} \text {. }}$

EXAMPLE 1. A concrete example of a class of constraints satisfying the exterior condition and entering in the applications to kinetically constrained models is as follows. Fix a vertex $z \succ 0$ and let $\mathcal{L}_{0}=\left\{x \in \mathbb{Z}^{d}:\langle x, z\rangle=0\right\}$, where $\langle\cdot, \cdot\rangle$ is the usual scalar product and $x, z$ are treated as vectors in $\mathbb{R}^{d}$. For $j \in \mathbb{Z}$, let $\mathcal{L}_{j}=\mathcal{L}_{0}+j \delta \vec{z}$ where $\delta=\sup \left\{\delta^{\prime}>0:\left(\mathcal{L}_{0}+\delta^{\prime} \vec{z}\right) \cap \mathbb{Z}^{d}=\varnothing\right\}$ (cf. Figure 1) and let $V_{n}=\bigcup_{j=-\infty}^{n} \mathcal{L}_{j}$. The above construction defines the exhausting collection of subsets $\left\{V_{n}\right\}_{n \in \mathbb{Z}}$.

Let now $G \subset S$ be an single-site event and let $\mathcal{U}=\left(U_{1}, \ldots, U_{m}\right)$ be a finite family of finite subsets of the half-space $\left\{x \in \mathbb{Z}^{d}:\langle x, z\rangle>0\right\}=\bigcup_{j=1}^{\infty} \mathcal{L}_{j}$. Then we define $c_{0}(\omega)$ as the indicator of the event that there exists $U \in \mathcal{U}$ such that $\omega_{x} \in G$

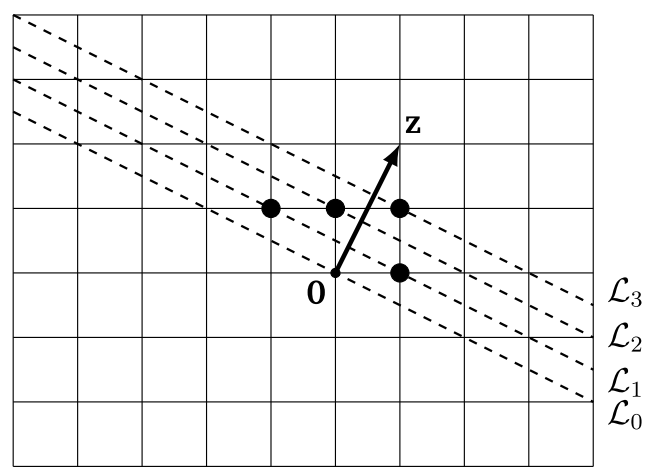

FIG. 1. An example in two dimensions of a constraint satisfying the exterior condition w.r.t. a sequence of increasing half-spaces. Only the shells $\left\{\mathcal{L}_{n}\right\}_{n=0}^{3}$ are drawn. The constraint $c_{0}$ requires that the restriction of the configuration $\omega$ to each one of the four vertices around the origin (black dots) belongs to a certain subset $G \subset S$. 
for all $x \in U$. The constraint $c_{x}$ at any other vertex $x$ is obtained by translating the above construction by $x$. For example, in $d=2$ one could take $S=\{0,1\}, G=\{0\}$, $z=(1,1), m=1$ and $U=\{(0,1),(1,0)\}$, a case known as the North-East model (cf., e.g., [8]).

2.4. Poincaré inequality. For simplicity, we state our main result directly for the infinite lattice $\mathbb{Z}^{d}$. There is also a finite-volume version in a box $\Lambda \subset \mathbb{Z}^{d}$ which is proved exactly in the same way. In the sequel, given a set $V \subset \mathbb{Z}^{d}$, we will write $\operatorname{Var}(f)$ and $\operatorname{Var}_{V}(f)$ for the variances of a function $f \in L^{2}(\Omega, \mu)$ w.r.t. to $\mu$ and to $\mu\left(\cdot \mid\left\{\omega_{y}\right\}_{y \notin V}\right)$, respectively.

Let $\left\{c_{x}^{(i)}\right\}_{x \in \mathbb{Z}^{d}}, i=1, \ldots, k$, be a family of constraints with supports $\Delta_{x}^{(i)}$ and failure probabilities $\varepsilon_{x}^{(i)}$. For any nonempty $I \subset[k]$, let $\lambda_{I} \in(0,+\infty)$ be a positive weight, let $\varepsilon_{x}^{(I)}=\mu\left(\prod_{i \in I}\left(1-c_{x}^{(i)}\right)\right)$ and let $\Delta_{x}^{(I)}=\bigcup_{i \in I} \Delta_{x}^{(i)}$.

THEOREM 2. Assume that there exists a choice of $\left\{\lambda_{I}\right\}_{I \subset[k]}$ such that

$$
\left(\sum_{\substack{I \subset[k] \\ I \neq \varnothing}} \lambda_{I}\right) \sup _{z} \sum_{\substack{I \subset[k] \\ I \neq \varnothing}} \sum_{\substack{x \in \mathbb{Z}^{d} \\ x \cup \Delta_{x}^{(I)}} z} \lambda_{I}^{-1} \varepsilon_{x}^{(I)}<1 / 4 .
$$

Suppose in addition that there exists an exhausting family $\left\{V_{n}\right\}_{n=-\infty}^{\infty}$ of sets of $\mathbb{Z}^{d}$ such that, for any $i \in[k]$, the constraints $\left\{c_{x}^{(i)}\right\}_{x \in \mathbb{Z}^{d}}$ satisfy the exterior condition w.r.t. $\left\{V_{n}\right\}_{n=-\infty}^{\infty}$. Then, for any local (i.e., depending on finitely many variables) function $f: \Omega \mapsto \mathbb{R}$,

$$
\operatorname{Var}(f) \leq 4 \sum_{x} \mu\left(\left[\prod_{i=1}^{k} c_{x}^{(i)}\right] \operatorname{Var}_{x}(f)\right)
$$

REMARK 2.2. The right-hand side of (2.2) is the Dirichlet form of a special $\mathrm{KCM}$ on $\mathbb{Z}^{d}$ with constraints $c_{x}=\prod_{i=1}^{k} c_{x}^{(i)}$ (see Section 4.1). Thus (2.2) says that the relaxation time of the above process (see Definition 4.2) is smaller than 4.

REMARK 2.3. It is easy to construct examples of constraints for which the exterior condition is violated and the right-hand side of (2.2) is zero for a suitable local function $f$. Take for instance $S=\{0,1\}, d=2$ and $c_{x}$ the indicator of the event that at least three nearest neighbours of $x$ are in the zero state. In this case, there does not exist an exhausting family $\left\{V_{n}\right\}_{n=1}^{\infty}$ such that the constraints satisfy the exterior condition w.r.t. $\left\{V_{n}\right\}_{n=1}^{\infty}$. Furthermore, if we let $f(\omega)=\omega_{0} \omega_{\vec{e}_{1}} \omega_{\vec{e}_{1}}+\vec{e}_{2} \omega_{\vec{e}_{2}}$ then $c_{x}(\omega) \operatorname{Var}_{x}(f)=0$ for all $\omega$ and all $x \in \mathbb{Z}^{d}$ while $\operatorname{Var}(f)>0$. Therefore, for this choice of $c_{x}$, inequality (2.2) does not hold for all local functions and the $\mathrm{KCM}$ with constraint $c_{x}$ has infinite relaxation time (see Remark 2.2). We stress that, however, the fact that the constraints satisfy the exterior condition is not a necessary condition in order for (2.2) to hold. See the following remark for further explanations. 
REMARK 2.4. For certain applications, the following monotonicity property turns out to be useful. Suppose that $\left\{c_{x}^{(i)}\right\}_{x \in \mathbb{Z}^{d}, i \in[k]}$ satisfy the condition of the theorem and let $\left\{\hat{c}_{x}^{(i)}\right\}_{x \in \mathbb{Z}^{d}, i \in[k]}$ be another family of constraints which are dominated by the first ones in the sense that $c_{x}^{(i)} \leq \hat{c}_{x}^{(i)}$ for all $i, x$. Then clearly (2.2) holds for all local functions with $c_{x}^{(i)}$ replaced bt $\hat{c}_{x}^{(i)}$ even if the latter does not satisfy the exterior condition. As an example take $S=\{0,1\}, k=1$ and $\hat{c}_{x}$ the constraint that at least two neighbours of $x$ are in the zero state (namely the constraint of FA-2f model, see Section 4.1) and $c_{x}$ the same but restricted to the neighbours of the form $x+\vec{e}_{i}, i \in[d]$.

Proof of TheOREM 2. We first treat the case of a single constraint $k=1$. After that we will explain how to generalize the argument to $k>1$ constraints. We begin with a simple result.

\section{LEMMA 2.5. For any local function $f$,}

$$
\operatorname{Var}(f) \leq \sum_{x} \mu\left(\operatorname{Var}_{x}\left(\mu_{\operatorname{Ext}_{x}}(f)\right)\right)
$$

Proof OF THE LEMmA. Let $\left\{V_{i}\right\}_{i=-\infty}^{\infty}$ be the exhausting family of sets w.r.t. which all the constraints satisfy the exterior condition, let $\mathcal{L}_{i}=V_{i} \backslash V_{i-1}$ be the corresponding $i$ th-shell and assume w.l.o.g. that the support of $f$ is contained in $\bigcup_{i=0}^{n} \mathcal{L}_{i}$. Let finally $\Lambda_{j}=\bigcup_{i=n-j}^{n} \mathcal{L}_{i}, j \leq n$. Using the formula for conditional variance together with the fact that $\mu$ is a product measure, we get

$$
\begin{aligned}
\operatorname{Var}(f) & =\mu\left(\operatorname{Var}_{\Lambda_{0}}(f)\right)+\operatorname{Var}\left(\mu_{\Lambda_{0}}(f)\right) \\
& =\mu\left(\operatorname{Var}_{\Lambda_{0}}(f)\right)+\mu\left(\operatorname{Var}_{\Lambda_{1}}\left[\mu_{\Lambda_{0}}(f)\right]\right)+\operatorname{Var}\left(\mu_{\Lambda_{1}}\left[\mu_{\Lambda_{0}}(f)\right]\right) \\
& \vdots \\
& =\mu\left(\operatorname{Var}_{\Lambda_{0}}[f]\right)+\sum_{j=0}^{n-1} \mu\left(\operatorname{Var}_{\Lambda_{j+1}}\left[\mu_{\Lambda_{j}}(f)\right]\right) .
\end{aligned}
$$

Recall now the standard inequality valid for any product probability measure $v=$ $v_{1} \otimes v_{2}$ :

$$
\operatorname{Var}_{v}(f) \leq v\left(\operatorname{Var}_{v_{1}}(f)\right)+v\left(\operatorname{Var}_{v_{2}}(f)\right)
$$

If we apply the inequality to $\operatorname{Var}_{\Lambda_{j+1}}\left[\mu_{\Lambda_{j}}(f)\right]$ and observe that $\mu_{\Lambda_{j}}(f)$ does not depend on the variables in $\Lambda_{j}$, we get immediately

$$
\begin{aligned}
\mu\left(\operatorname{Var}_{\Lambda_{j+1}}\left[\mu_{\Lambda_{j}}(f)\right]\right) & \leq \sum_{x \in \Lambda_{j+1} \backslash \Lambda_{j}} \mu\left(\operatorname{Var}_{x}\left(\mu_{\Lambda_{j}}(f)\right)\right) \\
& =\sum_{x \in \Lambda_{j+1} \backslash \Lambda_{j}} \mu\left(\operatorname{Var}_{x}\left(\mu_{\mathrm{Ext}_{x}}(f)\right)\right) .
\end{aligned}
$$


Analogously,

$$
\mu\left(\operatorname{Var}_{\Lambda_{0}}[f]\right) \leq \sum_{x \in \Lambda_{0}} \mu\left(\operatorname{Var}_{x}(f)\right)=\sum_{x \in \Lambda_{0}} \mu\left(\operatorname{Var}_{x}\left(\mu_{\text {Ext }_{x}}(f)\right)\right),
$$

because $\mu_{\operatorname{Ext}_{x}}(f)=f$ for any $x \in \Lambda_{0}$. The proof of the claim is complete.

We can now prove the theorem for $k=1$ and the starting point is (2.3). We begin by examining a generic term $\mu\left(\operatorname{Var}_{x}\left(\mu_{\operatorname{Ext}_{x}}(f)\right)\right)$ for which we write

$$
\mu_{\mathrm{Ext}_{x}}(f)=\mu_{\mathrm{Ext}_{x}}\left(c_{x} f\right)+\mu_{\mathrm{Ext}_{x}}\left(\left[1-c_{x}\right] f\right),
$$

so that

$$
\operatorname{Var}_{x}\left(\mu_{\operatorname{Ext}_{x}}(f)\right) \leq 2 \operatorname{Var}_{x}\left(\mu_{\operatorname{Ext}_{x}}\left(c_{x} f\right)\right)+2 \operatorname{Var}_{x}\left(\mu_{\operatorname{Ext}_{x}}\left(\left[1-c_{x}\right] f\right)\right) .
$$

Since $c_{x}(\omega)$ does not depend on $\omega_{x}$, the convexity of the variance implies that the first term in the above right-hand side satisfies

$$
\operatorname{Var}_{x}\left(\mu_{\mathrm{Ext}_{x}}\left(c_{x} f\right)\right) \leq \mu_{\mathrm{Ext}_{x}}\left(\operatorname{Var}_{x}\left(c_{x} f\right)\right)=\mu_{\mathrm{Ext}_{x}}\left(c_{x} \operatorname{Var}_{x}(f)\right)
$$

We now turn to the analysis of the more complicated second term in the right-hand side of (2.4).

$$
\begin{aligned}
\operatorname{Var}_{x} & \left(\mu_{\operatorname{Ext}_{x}}\left(\left(\left[1-c_{x}\right] f\right)\right)\right. \\
& =\operatorname{Var}_{x}\left(\mu_{\operatorname{Ext}_{x}}\left(\left[1-c_{x}\right]\left(f-\mu_{\operatorname{Ext}_{x} \cup\{x\}}(f)+\mu_{\operatorname{Ext}_{x} \cup\{x\}}(f)\right)\right)\right) \\
& =\operatorname{Var}_{x}\left(\mu_{\operatorname{Ext}_{x}}\left(\left[1-c_{x}\right] g\right)\right),
\end{aligned}
$$

where $g:=f-\mu_{\operatorname{Ext}_{x} \cup\{x\}}(f)$ and we used the fact that $\operatorname{Var}_{x}\left(\mu_{\operatorname{Ext}_{x}}\left(\left[1-c_{x}\right] \times\right.\right.$ $\left.\left.\mu_{\operatorname{Ext}_{x} \cup\{x\}}(f)\right)\right)=0$.

Recall now that the constraint $c_{x}$ depends only on $\left\{\omega_{y}\right\}_{y \in \Delta_{x}}$ with $\Delta_{x} \subset \operatorname{Ext}_{x}$. Thus

$$
\mu_{\operatorname{Ext}_{x}}\left(\left[1-c_{x}\right] g\right)=\mu_{\operatorname{Ext}_{x}}\left(\left[1-c_{x}\right] \mu_{\operatorname{Ext}_{x} \backslash \Delta_{x}}(g)\right)
$$

and a Schwarz inequality then gives

$$
\begin{aligned}
\operatorname{Var}_{x}\left(\mu_{\operatorname{Ext}_{x}}\left(\left[1-c_{x}\right] g\right)\right) & \leq \mu_{x}\left(\left(\mu_{\operatorname{Ext}_{x}}\left(\left(1-c_{x}\right) \mu_{\operatorname{Ext}_{x} \backslash \Delta_{x}} g\right)\right)^{2}\right) \\
& \leq \varepsilon_{x} \mu_{\operatorname{Ext}_{x} \cup\{x\}}\left(\left[\mu_{\operatorname{Ext}_{x} \backslash \Delta_{x}}(g)\right]^{2}\right) .
\end{aligned}
$$

Next, we note that

$$
\begin{aligned}
\mu_{\operatorname{Ext}_{x} \cup\{x\}}\left(\left[\mu_{\operatorname{Ext}_{x} \backslash \Delta_{x}}(g)\right]^{2}\right) & =\mu_{x \cup \Delta_{x}}\left(\mu_{\operatorname{Ext}_{x} \backslash \Delta_{x}}(g)^{2}\right) \\
& =\operatorname{Var}_{x \cup \Delta_{x}}\left(\mu_{\operatorname{Ext}_{x} \backslash \Delta_{x}}(g)\right),
\end{aligned}
$$


where we used the fact that $\mu_{x \cup \Delta_{x}}\left(\mu_{\operatorname{Ext}_{x} \backslash \Delta_{x}}(g)\right)=\mu_{\operatorname{Ext}_{x} \cup\{x\}}(g)=0$ by the definition of $g$. Then by using (2.3), (2.5) and (2.6) we get

$$
\begin{aligned}
\operatorname{Var}_{x}\left(\mu_{\operatorname{Ext}_{x}}\left(\left[1-c_{x}\right] g\right)\right) & \leq \varepsilon_{x} \sum_{z \in x \cup \Delta_{x}} \mu_{x \cup \Delta_{x}}\left(\operatorname{Var}_{z}\left(\mu_{\operatorname{Ext}_{z}}\left[\mu_{\operatorname{Ext}_{x} \backslash \Delta_{x}}(g)\right]\right)\right) \\
& \leq \varepsilon_{x} \sum_{z \in x \cup \Delta_{x}} \mu_{\operatorname{Ext}_{x} \cup\{x\}}\left(\operatorname{Var}_{z}\left(\mu_{\operatorname{Ext}_{z}}(g)\right)\right. \\
& =\varepsilon_{x} \sum_{z \in x \cup \Delta_{x}} \mu_{\operatorname{Ext}_{x} \cup\{x\}}\left(\operatorname{Var}_{z}\left(\mu_{\operatorname{Ext}_{z}}(f)\right),\right.
\end{aligned}
$$

where we use the convexity of the variance to obtain the second inequality.

In conclusion,

$$
\begin{aligned}
\sum_{x} \mu & \left(\operatorname{Var}_{x}\left(\mu_{\operatorname{Ext}_{x}}(f)\right)\right) \\
& \leq 2 \sum_{x} \mu\left(c_{x} \operatorname{Var}_{x}(f)\right)+2 \sum_{x} \varepsilon_{x} \sum_{z \in x \cup \Delta_{x}} \mu\left(\operatorname{Var}_{z}\left(\mu_{\operatorname{Ext}_{z}}(f)\right)\right) \\
& \leq 2 \sum_{x} \mu\left(c_{x} \operatorname{Var}_{x}(f)\right)+2\left[\sup _{z} \sum_{x: x \cup \Delta_{x} \ni z} \varepsilon_{x}\right] \sum_{z} \mu\left(\operatorname{Var}_{z}\left(\mu_{\operatorname{Ext}_{z}}(f)\right)\right) .
\end{aligned}
$$

If $\sup _{z} \sum_{x: x \cup \Delta_{x} \ni z} \varepsilon_{x} \leq 1 / 4$ we get

$$
\sum_{x} \mu\left(\operatorname{Var}_{x}\left(\mu_{\operatorname{Ext}_{x}}(f)\right)\right) \leq 4 \sum_{x} \mu\left(c_{x} \operatorname{Var}_{x}(f)\right)
$$

We now turn to the general case $k>1$. Let $c_{x}=\prod_{i} c_{x}^{(i)}$ and recall the definition of $\varepsilon_{x}^{(I)}$ and of $\Delta_{x}^{(I)}$ for any nonempty $I \subset[k]$. Let also $d_{x}^{(I)}=\prod_{i \in I}\left(1-c_{x}^{(i)}\right)$ so that $\varepsilon_{x}^{(I)}=\mu\left(d_{x}^{(I)}\right)$. Notice that (inclusion/exclusion formula)

$$
1-c_{x}=\sum_{\substack{I \subset[k] \\ I \neq \varnothing}}(-1)^{\operatorname{Parity}(I)+1} d_{x}^{(I)}=\sum_{\substack{I \subset[k] \\ I \neq \varnothing}}(-1)^{1+\operatorname{Parity}(I)} \sqrt{\lambda_{I}} d_{x}^{(I)} / \sqrt{\lambda_{I}} .
$$

Thus the delicate term $\operatorname{Var}_{x}\left(\mu_{\mathrm{Ext}_{x}}\left(\left(\left[1-c_{x}\right] f\right)\right)\right.$ in $(2.4)$ can be bounded from above using the Schwarz inequality by

$$
\left(\sum_{\substack{I \subset[k] \\ I \neq \varnothing}} \lambda_{I}\right) \sum_{\substack{I \subset[k] \\ I \neq \varnothing}} \lambda_{I}^{-1} \operatorname{Var}_{x}\left(\mu_{\mathrm{Ext}_{x}}\left(d_{x}^{(I)} f\right)\right)
$$

At this stage, we apply the steps leading to (2.7) to each term $\operatorname{Var}_{x}\left(\mu_{\operatorname{Ext}_{x}}\left(d_{x}^{(I)} f\right)\right)$ to get

$$
\begin{aligned}
& \operatorname{Var}_{x}\left(\mu_{\operatorname{Ext}_{x}}\left(\left(\left[1-c_{x}\right] f\right)\right)\right. \\
& \quad \leq\left(\sum_{\substack{I \subset[k] \\
I \neq \varnothing}} \lambda_{I}\right) \sum_{\substack{I \subset[k] \\
I \neq \varnothing}} \lambda_{I}^{-1} \varepsilon_{x}^{(I)} \sum_{\substack{z \in x \cup \Delta_{x}^{(I)}\\
}} \mu_{\operatorname{Ext}_{x} \cup\{x\}}\left(\operatorname{Var}_{z}\left(\mu_{\operatorname{Ext}_{z}}(f)\right) .\right.
\end{aligned}
$$


As in (2.8), we conclude that

$$
\begin{aligned}
\sum_{x} \mu\left(\operatorname{Var}_{x}\left(\mu_{\operatorname{Ext}_{x}}(f)\right)\right) & \\
\leq & 2 \sum_{x} \mu\left(c_{x} \operatorname{Var}_{x}(f)\right) \\
& +2\left(\sum_{\substack{I \subset[k] \\
I \neq \varnothing}} \lambda_{I}\right)\left(\sup _{z} \sum_{\substack{I \subset[k] \\
I \neq \varnothing}} \sum_{\substack{x \\
I \neq \Delta_{x}^{(I)}} z} \lambda_{I}^{-1} \varepsilon_{x}^{(I)}\right) \sum_{z} \mu\left(\operatorname{Var}_{z}\left(\mu_{\operatorname{Ext}_{z}}(f)\right)\right),
\end{aligned}
$$

which proves the theorem if

$$
\left(\sum_{\substack{I \subset[k] \\ I \neq \varnothing}} \lambda_{I}\right)\left(\sup _{z} \sum_{\substack{I \subset[k] \\ I \neq \varnothing}} \sum_{\substack{x \\ I \neq \Delta_{x}^{(I)} \ni z}} \lambda_{I}^{-1} \varepsilon_{x}^{(I)}\right) \leq 1 / 4 .
$$

2.5. An application within supercritical percolation in two dimensions. In this section, we restrict ourselves to the case in which the single site probability space $(S, \hat{\mu})$ coincides with $(\{0,1\}, B(p))$ and the lattice dimension is equal to two. Given $\omega \in \Omega:=\Omega_{\mathbb{Z}^{2}}$, we will say that $x \in \mathcal{C}(\omega):=\left\{x \in \mathbb{Z}^{2}: \omega_{x}=0\right\}$ belongs to an infinite cluster of zeros if the connected (w.r.t. to the graph structure of $\mathbb{Z}^{2}$ ) component of $\mathcal{C}(\omega)$ containing $x$ is unbounded. It is well known that there exists $p_{c} \in(0,1)$ such that

$$
\theta(p):=\mu(\text { the origin belongs to an infinite cluster) }
$$

is positive iff $p<p_{c}$ and that moreover there exists $\mu$-a.s. a unique unbounded component of $\mathcal{C}(\omega)$ The conjectured threshold $p_{c}$ is approximately $1-p_{c} \approx 0.59$ [21]. Fix $x \in \mathbb{Z}^{2}, \omega \in \Omega$ and let $\bar{\omega}$ be the configuration obtained from $\omega$ by setting to 1 site $x$, namely $\bar{\omega}_{x}=1$ and $\bar{\omega}_{y}=\omega_{y}$ for $y \neq x$. We let $c_{x}^{\infty}(\omega)=1$ if at least two nearest neighbors of $x$ belong to an infinite cluster of zeros in the configuration $\bar{\omega}$, $c_{x}^{\infty}(\omega)=0$ otherwise.

THEOREM 2.6. There exists $p_{0} \in\left(0, p_{c}\right)$ such that for any $p \leq p_{0}$ and any local function $f$

$$
\operatorname{Var}(f) \leq 4 \sum_{x} \mu\left(c_{x}^{\infty} \operatorname{Var}_{x}(f)\right)
$$

REMARK 2.7. It follows in particular that, for all $p$ sufficiently small, the kinetically constrained model with constraints $\left\{c_{x}^{\infty}\right\}_{x \in \mathbb{Z}^{2}}$ (cf. Section 4.1) has its relaxation time bounded by 4 .

PROOF. We will make use of the following standard construction for supercritical percolation [12]. Let $\ell_{n}=2^{n}$ and define $R_{n}$ to be a rectangle of the form 


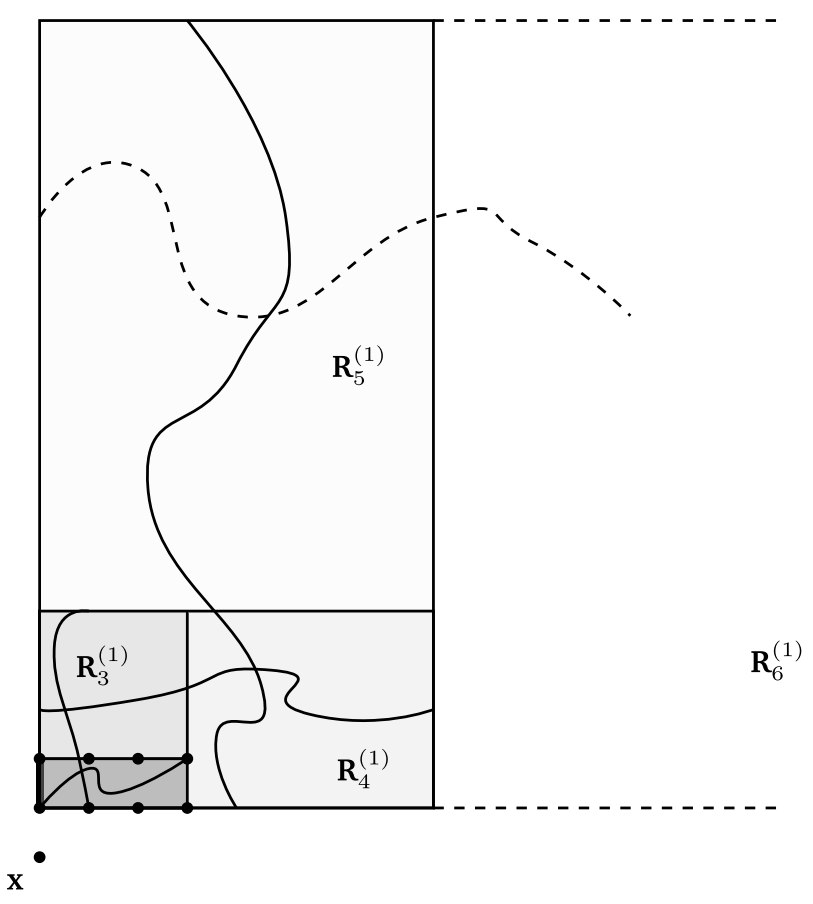

FIG. 2. A drawing of the first five rectangles $\left\{R_{n}^{(1)}+x\right\}_{n=1}^{5}$ together with a pictorial representation of the hard crossings of zeros (the solid lines) required by the auxiliary constraint $c_{x}^{(n, 1)}$. The dashed curved line represents a piece of the hard crossing for the next rectangle $R_{6}^{(1)}+x$ (the two horizontal dashed lines). Notice that each rectangle has its leftmost lowermost vertex always at $x+\vec{e}_{2}$ and that the first rectangle $R_{1}^{(1)}$ consists of only two vertices, $x+\vec{e}_{2}$ and $x+2 \vec{e}_{2}$.

either $\left[\ell_{n}\right] \times\left[\ell_{n-1}\right]$ or $\left[\ell_{n-1}\right] \times\left[\ell_{n}\right]$ according to whether $n$ is even or odd. We will also denote by $R_{n}^{(1)}\left(R_{n}^{(2)}\right)$ the rectangle obtained by translating $R_{n}$ by the vector $-\vec{e}_{1}\left(-\vec{e}_{2}\right)$ (see Figure 2). With the help of the families $\left\{R_{n}^{(1)}, R_{n}^{(2)}\right\}_{n \in \mathbb{N}}$, we finally introduce a new family of constraints as follows.

Let a path $\gamma$ in $\mathbb{Z}^{d}$ of length $|\gamma|:=k$ be an ordered sequence of $k$ vertices of $\mathbb{Z}^{2}$ such that two consecutive sites are nearest neighbors of each other. For $i=1,2$, let $c_{x}^{(n, i)}$ be the indicator function of the event that inside the rectangle $R_{n}^{(i)}+x$ there exists an hard crossing, that is, a path $\gamma=\left(x^{(1)}, \ldots, x^{(m)}\right)$ joining the two opposite shortest sides such that $\omega_{x^{(j)}}=0$ for all $j \in[m]$. Let also $c_{x}^{(0)}$ be the indicator of the event that $\omega_{x+\vec{e}_{1}}=\omega_{x+\vec{e}_{2}}=0$. Notice that, by construction, the above constraints satisfy the exterior condition 2.1 w.r.t. to the half-spaces defined in Example 1 with $z=(1,1)$. Moreover, it is easy to check that

$$
c_{x}^{(0)} \prod_{n=1}^{\infty} c_{x}^{(n, 1)} c_{x}^{(n, 2)} \leq c_{x}^{\infty} \quad \forall x,
$$


so that it is enough to prove the constrained Poincare inequality (2.9) with $c_{x}^{\infty}$ replaced by $c_{x}^{(0)} \prod_{n=1}^{\infty} c_{x}^{(n, 1)} c_{x}^{(n, 2)}$. More precisely, we will prove that, for any $k \in \mathbb{N}$ and any local function $f$,

$$
\operatorname{Var}(f) \leq 4 \sum_{x} \mu\left(c_{x}^{(0)} \prod_{n=1}^{k} c_{x}^{(n, 1)} c_{x}^{(n, 2)} \operatorname{Var}_{x}(f)\right)
$$

The theorem will then follow by taking the limit $k \rightarrow+\infty$ and using (2.10). In order to prove (2.11), we want to apply Theorem 2 which in turn requires finding a family of weights $\left\{\lambda_{I}\right\}_{I \subset[k] \cup\{0\}}$ satisfying (2.1). A standard Peierls' argument implies that, for all $p$ small enough,

$$
\mu\left(1-c_{x}^{(n, i)}\right) \leq e^{-m(p) \ell_{n}},
$$

with $\lim _{p \rightarrow 0} m(p)=+\infty$. In particular, recalling the definition of $\varepsilon_{x}^{(I)}$ and $\Delta_{x}^{(I)}$ from Section 2.4, we have the following bounds:

$$
\begin{aligned}
& \varepsilon_{x}^{(I)} \leq e^{-m(p) \ell_{n(I)}}, \quad\left|\Delta_{x}^{(I)}\right| \leq 3 \ell_{n(I)}^{2} \quad \text { if } n(I):=\max \{i \in I\}>0, \\
& \varepsilon_{x}^{(I)} \leq 2 p, \quad\left|\Delta_{x}^{(I)}\right| \leq 2 \quad \text { otherwise. }
\end{aligned}
$$

Let now $\lambda_{I}=e^{-\frac{m(p)}{2} \ell_{n(I)}}$ if $I \neq\{0\}$ and $\lambda_{I}=\sqrt{p}$ if $I=\{0\}$. With this choice, it is easy to check that there exists $p_{0}$ independent of $k$ such that for $p<p_{0}$,

$$
\sum_{I \subset[k] \cup\{0\}} \lambda_{I} \leq 1 / 2
$$

and

$$
\begin{aligned}
\sup _{z} \sum_{\substack{I \subset[k] \cup\{0\} \\
I \neq \varnothing}} \sum_{\substack{x \in \mathbb{Z}^{d} \\
x \cup \Delta_{x}^{(I)} \ni z}} \lambda_{I}^{-1} \varepsilon_{x}^{(I)} & \leq 3 \sum_{\substack{I \subset[k] \cup\{0\} \\
I \neq \varnothing, I \neq\{0\}}} \ell_{n(I)}^{2} e^{-\frac{m(p)}{2} \ell_{n(I)}}+4 \sqrt{p} \\
& \leq 3 \sum_{n=1}^{\infty} 2^{n} 4^{n} e^{-\frac{m(p)}{2} \ell_{n}}+4 \sqrt{p} \leq 1 / 4 .
\end{aligned}
$$

In conclusion, (2.1) holds for all small enough $p$ independent of $k$ and the theorem follows.

\section{A general approach to prove a Poincaré inequality for kinetically con-} strained spin models. In this section, we start from the general constrained Poincaré inequality proved in Theorem 2 to develop a quite robust and general scheme proving a special kind of Poincaré constrained inequality (cf. Theorem 3.2 and Corollary 3.9) that will be crucial to determine a sharp upper bound on the mean infection time of KCM. Concrete and successful applications to basic kinetically constrained models (cf. Theorem 4.4) will be given in the next section. 
The starting point of our approach is the definition of good and super-good single site events. Given two events $G_{1}, G_{2}$ in the probability space $(S, \hat{\mu})$, let $p_{1}:=\hat{\mu}\left(G_{1}\right)$ and $p_{2}:=\hat{\mu}\left(G_{2}\right)$. We will assume that $G_{1}$ is very likely while $G_{2}$ is very unlikely. In the sequel, we will refer to $G_{1}$ and $G_{2}$ as the good and supergood events, respectively. In the applications, $G_{2}$ will guarantee the presence of a certain bootstrap critical droplet, while $G_{1}$ will guarantee the presence of enough infected vertices to allow a critical droplet to grow.

DEFINITION 3.1 (Good and super-good paths). Given $\omega \in \Omega=S^{\mathbb{Z}^{d}}$, we will say that a vertex $x$ is good if $\omega_{x} \in G_{1}$ and super-good if $\omega_{x} \in G_{2}$. We will say that a path $\gamma=\left(x^{(1)}, \ldots, x^{(k)}\right)$ is a good path for $\omega$ if each vertex in $\gamma$ is good. A path will be called super-good if it is good and it contains at least one super-good vertex.

Before stating the main result, we need a last notion. For any mapping $G_{1} \stackrel{\oplus}{\mapsto}$ $G_{2}$, let

$$
\lambda_{\Phi}=\max _{\sigma \in G_{2}} \sum_{\sigma^{\prime} \in G_{1}: \Phi\left(\sigma^{\prime}\right)=\sigma} \frac{\hat{\mu}\left(\sigma^{\prime}\right)}{\hat{\mu}(\sigma)},
$$

and, for any $\omega$ such that $\omega_{x} \in G_{1}$, let $\Phi^{(x)}: \Omega \mapsto \Omega$ be given by

$$
\Phi^{(x)}(\omega)_{z}:= \begin{cases}\Phi\left(\omega_{x}\right) & \text { if } z=x \\ \omega_{z} & \text { otherwise }\end{cases}
$$

THEOREM 3.2. There exist $\delta \ll 1$ and $c>0$ such that, for any $G_{1} \stackrel{\Phi}{\mapsto} G_{2}$ and all $p_{1}, p_{2}$ with $\max \left(p_{2},\left(1-p_{1}\right) \log \left(1 / p_{2}\right)^{2}\right) \leq \delta$, the following holds:

$$
\begin{aligned}
\operatorname{Var}(f) \leq & c\left(\lambda_{\Phi} p_{2}^{-4}\right)^{d}\left[\sum_{x} \mu\left(\left[\prod_{i \in[d]} \mathbb{1}_{\left\{\omega_{x+\vec{e}_{i}} \in G_{2}\right\}}\right] \operatorname{Var}_{x}(f)\right)\right. \\
& \left.+\sum_{x, y: d_{1}(x, y)=1} \mu\left(\mathbb{1}_{\left\{\omega_{x} \in G_{1}, \omega_{y} \in G_{2}\right\}}\left[f\left(\Phi^{(x)}(\omega)\right)-f(\omega)\right]^{2}\right)\right] .
\end{aligned}
$$

REMARK 3.3. We could have stated Theorem 3.2 in a more general form in which the constraint $\prod_{i \in[d]} \mathbb{1}_{\left\{\omega_{x+\vec{e}_{i}} \in G_{2}\right\}}$, appearing in the first term in the righthand side of (3.3), is replaced by $\prod_{y \in A+x} \mathbb{1}_{\left\{\omega_{y} \in G_{2}\right\}}$, where $A \subset \mathrm{Ext}_{0}$ is some finite set whose cardinality is independent of $p_{1}, p_{2}$. For example, in two dimensions $A$ could be $\left\{\vec{e}_{1}\right\} \cup\left\{\vec{e}_{2}+\vec{e}_{1}\right\} \cup\left\{\vec{e}_{2}\right\} \cup \cdots \cup\left\{\vec{e}_{2}-m \vec{e}_{1}\right\}$. For future applications [24], the freedom given by the choice of the set $A$ will be quite crucial. The proof in this slightly more general case is identical to the one given below. The same applies for the developments discussed in Section 3.1. 
The first term in the right-hand side of (3.3) is a constrained Dirichlet form $\mathcal{D}(f)$ as in the r.h.s. of (2.2), with constraints $c_{x}:=\prod_{i \in[d]} \mathbb{1}_{\left\{\omega_{x+\vec{e}_{i}} \in G_{2}\right\}}$. These constraints satisfy the exterior condition w.r.t. the half-spaces defined in Example 1 with $z=(1, \ldots, 1)$ but, at the same time, they are very unlikely [recall that $\left.\hat{\mu}\left(G_{2}\right) \ll 1\right]$ so that we cannot apply directly Theorem 2 to our setting. Moreover, the fact that the $\left\{c_{x}\right\}$ are unlikely implies that a Poincare inequality of the form $\operatorname{Var}(f) \leq C \mathcal{D}(f)$ for all local $f$ and some finite constant $C$ cannot hold. To see that, take for instance $\left\{f_{n}\right\}_{n=1}^{\infty}$ to be a sequence of local functions approximating the indicator of the event that the origin belongs to an infinite oriented cluster of not super-good vertices. In other words, there exists a infinite path $\gamma=\left(x^{(1)}, \ldots, x^{(k)}, \ldots\right)$ starting at the origin such that $x^{(i)} \prec x^{(i+1)}$ and $\omega_{x^{(i)}} \notin G_{2}$ for all $i$. Thus the second term in the right-hand side of (3.3) plays an important role.

Our approach is first to prove a different kind of constrained Poincaré inequality (cf. Proposition 3.4) in which the term in (3.3) involving $\Phi$ is missing and the constraint $c_{x}$ above is replaced by the weaker (and very likely) constraint that for all $i \in[d]$ there exists a super-good path $\gamma^{(i)}$ in $\mathbb{Z}^{2} \backslash\{x\}$ starting at $x+\vec{e}_{i}$ and of length not larger than $1 / p_{2}^{2}$. Second (cf. Lemma 3.5), using repeatedly the mapping $\Phi^{x}$ for each $x \in \gamma^{(i)}$ starting at the super-good vertex of $\gamma^{(i)}$, we "bring" the supergood vertex of $\gamma^{(i)}$ at $x+\vec{e}_{i}$. In doing that, we pay a cost which is embodied in the second term in the right-hand side of (3.3).

Proof of TheOrem 3.2. In what follows, we assume that we have fixed some mapping $G_{1} \stackrel{\Phi}{\mapsto} G_{2}$. We begin by proving the first step of the roadmap just described.

PROPOSITION 3.4. There exists $\delta \ll 1$ such that, for all $p_{1}$, p $p_{2}$ satisfying $\max \left(p_{2},\left(1-p_{1}\right) \log \left(1 / p_{2}\right)^{2}\right) \leq \delta$, the following holds. Let $\mathbb{1}_{x}$ be the indicator of the event that $\forall i \in[d]$ there exists a super-good path $\gamma^{(i)}$ of length at most $1 / p_{2}^{2}$ starting at $x+\vec{e}_{i}$. Then, for any local $f$,

$$
\operatorname{Var}(f) \leq 4 \sum_{x} \mu\left(\mathbb{1}_{x} \operatorname{Var}_{x}(f)\right)
$$

PROOF OF THE PROPOSITION. In what follows, all the auxiliary constraints that we will need to introduce will satisfy the exterior condition w.r.t. the exhausting family of half-spaces defined in Example 1 with $z=(1, \ldots, 1)$.

Let $\ell=\left\lfloor 2 \log \left(1 / p_{2}\right)\right\rfloor, L=\left\lfloor e^{\ell}\right\rfloor$ and let us define two families of constraints $\left\{c_{x}^{(1)}, c_{x}^{(2)}\right\}_{x \in \mathbb{Z}^{d}}$ as follows:

$$
c_{x}^{(1)}= \begin{cases}1 & \text { if for all } i \in[d] \text { and all } k \in[\ell] \text { the vertex } x+k \vec{e}_{i} \text { is good, } \\ 0 & \text { otherwise }\end{cases}
$$




$$
c_{x}^{(2)}= \begin{cases}1 & \begin{array}{l}
\text { if for all } i \in[d] \exists \text { a super-good path in } \operatorname{Ext}_{x} \text { of length at most } L \\
\text { starting in the set }\left\{x+\vec{e}_{i}, \ldots, x+\ell \vec{e}_{i}\right\}, \\
0 \quad \text { otherwise. }
\end{array}\end{cases}
$$

Notice that $c_{x}^{(1)} c_{x}^{(2)} \leq \mathbb{1}_{x}$. In order to apply Theorem 2 to the above constraints, we need to verify the key condition (2.1). For this purpose, we begin to observe that the corresponding supports satisfy $\Delta_{x}^{(1)} \subset \bigcup_{i=1}^{d}\left\{x+\vec{e}_{i}, \ldots, x+\ell \vec{e}_{i}\right\}$ and $\Delta_{x}^{(2)} \subset$ $\left\{y \in \mathbb{Z}^{d}: d_{1}(x, y) \leq \ell+L\right\}$. In particular, there exists a numerical constant $\hat{\delta}$ such that the condition for the validity of Theorem 2 holds if

$$
d \ell \mu\left(1-c_{x}^{(1)}\right)+(\ell+L)^{d}\left(\mu\left(\left(1-c_{x}^{(2)}\right)\right)+\mu\left(\left(1-c_{x}^{(1)}\right)\left(1-c_{x}^{(2)}\right)\right)\right) \leq \hat{\delta} .
$$

A simple union bound proves that $\mu\left(1-c_{x}^{(1)}\right) \leq d \ell\left(1-p_{1}\right)$, while standard supercritical percolation bounds valid for large enough values of $p_{1}$ prove that

$$
\mu\left(\left(1-c_{x}^{(1)}\right)\left(1-c_{x}^{(2)}\right)\right) \leq \mu\left(1-c_{x}^{(2)}\right) \leq d\left(e^{-c \log \left(1 /\left(1-p_{1}\right)\right) \ell}+\left(1-p_{2}\right)^{L}\right)
$$

for some constant $c>0$. Fix, for example, the first direction. The probability that none of the vertices $x+\vec{e}_{1}, \ldots, x+\ell \vec{e}_{1}$ belong to an infinite good path in $\mathrm{Ext}_{x}$ is exponentially small in $\ell$ while the probability that a given path of length $L$ is super-good conditionally on being good is at least $1-\left(1-p_{2}\right)^{L}$. It is now immediate to verify that given $\hat{\delta}>0$ there exists $\delta>0$ small enough such that $\max \left(p_{2},\left(1-p_{1}\right) \log \left(1 / p_{2}\right)^{2}\right) \leq \delta$ implies (3.5).

Notice that so far the mapping $\Phi$ played no role. We will now use it in order to bound a generic term $\mu\left(\mathbb{1}_{x} \operatorname{Var}_{x}(f)\right)$ appearing in (3.4). Without loss of generality, we only treat the case $x=0$.

LEMMA 3.5. In the same setting of Theorem 3.2, there exists $c>0$ independent of $p_{1}, p_{2}, \Phi$ such that

$$
\begin{aligned}
\mu\left(\mathbb{1}_{0} \operatorname{Var}_{0}(f)\right) \leq & c\left(\lambda_{\Phi} p_{2}^{-2}\right)^{d}\left[\mu\left(\left[\prod_{i \in[d]} \mathbb{1}_{\left\{\omega_{\vec{e}_{i}} \in G_{2}\right\}}\right] \operatorname{Var}_{0}(f)\right)\right. \\
& \left.+\sum_{\substack{x, y: \in \Lambda \backslash\{0\} \\
d_{1}(x, y)=1}} \mu\left(\mathbb{1}_{\left\{\omega_{x} \in G_{1}, \omega_{y} \in G_{2}\right\}}\left[f\left(\Phi^{(x)}(\omega)\right)-f(\omega)\right]^{2}\right)\right],
\end{aligned}
$$

where $\Lambda$ is the box centered at the origin of side $2\left\lfloor 1 / p_{2}^{2}\right\rfloor$.

By combining together Lemma 3.5 and Proposition 3.4, we get the statement of the theorem.

PROOF OF LEMMA 3.5. Recall that $\mathbb{1}_{0}$ is the indicator of the event $\bigcap_{i \in[d]} S G_{i}$, where $S G_{i}$ is the event that there exists a super-good path $\gamma^{(i)}$ in $\mathbb{Z}^{2} \backslash\{0\}$ of length at most $L \equiv 1 / p_{2}^{2}$ starting at $\vec{e}_{i}$. Clearly, $S G_{i}$ is identical to the event that there exists $\gamma=\left(x^{(1)}, \ldots, x^{(L)}\right) \subset \mathbb{Z}^{2} \backslash\{0\}$, such that: 
- each vertex $x^{(j)}$ appears exactly once (i.e., the path is simple) and $x^{(1)}=\vec{e}_{i}$,

- there exist $n \leq L$ such that $x^{(n)}$ is super-good,

- all the vertices $x^{(j)}$ with $j \leq n$ are good.

Fix $i=1$ and let us order in some way the set $\mathcal{P}$ of simple paths in $\mathbb{Z}^{d} \backslash\{0\}$ of length $L$ starting at $\vec{e}_{1}$. For any $\omega \in \bigcap_{i \in[d]} S G_{i}$, let $\gamma^{*}$ be the smallest path in $\mathcal{P}$ satisfying the above set of conditions and let $v=v(\omega)$ be the index of the first super-good vertex in $\gamma^{*}$. Thus

$$
\mu\left(\mathbb{1}_{0} \operatorname{Var}_{0}(f)\right)=\sum_{\gamma \in \mathcal{P}} \sum_{n=1}^{L} \mu\left(\mathbb{1}_{\left\{\gamma^{*}=\gamma\right\}} \mathbb{1}_{\{v=n\}} \prod_{j=2}^{d} \mathbb{1}_{\left\{S G_{j}\right\}} F\right),
$$

where

$$
F(\omega):=\operatorname{Var}_{0}(f)(\omega)=\frac{1}{2} \sum_{\sigma, \sigma^{\prime} \in S} \hat{\mu}(\sigma) \hat{\mu}\left(\sigma^{\prime}\right)\left(f(\omega \otimes \sigma)-f\left(\omega \otimes \sigma^{\prime}\right)\right)^{2},
$$

where the notation $\omega \otimes \sigma$ denotes the configuration equal to $\sigma$ at $x=0$ and equal to $\omega$ elsewhere.

Given $\gamma=\left(x^{(1)}, \ldots, x^{(L)}\right) \in \mathcal{P}$ and $n \leq L$ together with $\omega \in \prod_{j \in[d]} S G_{j}$ such that $\gamma^{*}(\omega)=\gamma$ and $v(\omega)=n$, let $\Phi^{(i)}(\omega)$ be given by [recall (3.2)]

$$
\Phi^{(i)}(\omega)= \begin{cases}\Phi^{\left(x^{(i)}\right)}(\omega) & \text { if } i \leq n-1, \\ \omega & \text { if } i=n .\end{cases}
$$

Thus the mapping $\Phi^{(i)}, i \leq n-1$, makes the configuration $\omega$ super-good in $x^{(i)}$ and leaves it unchanged elsewhere. For $i=n$, the mapping $\Phi^{(n)}$ is the identity. With the above notation and using the Cauchy-Schwarz inequality, we get

$$
F(\omega) \leq 2 F\left(\Phi^{(1)}(\omega)\right)+4 \sum_{\sigma \in S} \hat{\mu}(\sigma)\left(f\left(\Phi^{(1)}(\omega) \otimes \sigma\right)-f(\omega \otimes \sigma)\right)^{2} .
$$

The first term in the right-hand side of (3.8) gives a contribution to the right-hand side of (3.7) not larger than

$$
2 \lambda_{\Phi} \mu\left(\mathbb{1}_{\left\{\omega_{\overrightarrow{\vec{e}_{1}}} \in G_{2}\right\}} \prod_{j=2}^{d} \mathbb{1}_{\left\{S G_{j}\right\}} \operatorname{Var}_{0}(f)\right) .
$$

Above, after the change of variable $\eta:=\Phi^{(1)}(\omega)$, we used (3.1) together with the obvious facts that $\eta$ is super-good at $\vec{e}_{1}$ and it belongs to $\prod_{j=2}^{d} \mathbb{1}_{S G_{j}}$. 
In order to bound from above the contribution of the second term in the righthand side of (3.8), we write

$$
\begin{aligned}
& \left(f\left(\Phi^{(1)}(\omega) \otimes \sigma\right)-f(\omega \otimes \sigma)\right)^{2} \\
& \quad=\left(\sum_{i=1}^{n-1}\left[f\left(\Phi^{(i+1)}(\omega) \otimes \sigma\right)-f\left(\Phi^{(i)}(\omega) \otimes \sigma\right)\right]\right)^{2} \\
& \quad \leq(n-1) \sum_{i=1}^{n-1}\left[f\left(\Phi^{(i+1)}(\omega) \otimes \sigma\right)-f\left(\Phi^{(i)}(\omega) \otimes \sigma\right)\right]^{2} \\
& \quad \leq L \sum_{i=1}^{n-1}\left[f\left(\Phi^{(i+1)}(\omega) \otimes \sigma\right)-f\left(\Phi^{(i)}(\omega) \otimes \sigma\right)\right]^{2} .
\end{aligned}
$$

In turn each summand is bounded from above by

$$
\begin{aligned}
& 2\left[f\left(\Phi^{(i+1)}\left(\Phi^{(i)}(\omega)\right) \otimes \sigma\right)-f\left(\Phi^{(i)}(\omega) \otimes \sigma\right)\right]^{2} \\
& \quad+2\left[f\left(\Phi^{(i+1)}(\omega) \otimes \sigma\right)-f\left(\Phi^{(i+1)}\left(\Phi^{(i)}(\omega)\right) \otimes \sigma\right)\right]^{2} .
\end{aligned}
$$

Using the fact that $\Phi^{(i+1)}\left(\Phi^{(i)}(\omega)\right)=\Phi^{(i)}\left(\Phi^{(i+1)}(\omega)\right)$, we see that both terms in the right-hand side above have a similar structure. We will therefore treat explicitly only the first one. Recalling that $\Lambda$ is the box centered at the origin with side $2\left\lfloor 1 / p_{2}^{2}\right\rfloor$, we get

$$
\begin{aligned}
& 2 L \mu\left(\sum_{\gamma \in \mathcal{P}} \sum_{n=1}^{L} \sum_{i=1}^{n-1} \mathbb{1}_{\left\{\gamma^{*}=\gamma\right\}} \mathbb{1}_{\{\nu=n\}} \prod_{j=2}^{d} \mathbb{1}_{\left\{S G_{j}\right\}}\right. \\
& \left.\times \sum_{\sigma \in S} \hat{\mu}(\sigma)\left[f\left(\Phi^{(i+1)}\left(\Phi^{(i)}(\omega)\right) \otimes \sigma\right)-f\left(\Phi^{(i)}(\omega) \otimes \sigma\right)\right]^{2}\right) \\
& =2 L \mu\left(\mathbb{1}_{S G_{1}} \prod_{j=2}^{d} \mathbb{1}_{\left\{S G_{j}\right\}} \sum_{i=1}^{\nu-1}\left[f\left(\Phi^{(i+1)}\left(\Phi^{(i)}(\omega)\right) \otimes \sigma\right)-f\left(\Phi^{(i)}(\omega) \otimes \sigma\right)\right]^{2}\right) \\
& \leq 2 L \sum_{\substack{x, y \in \Lambda \backslash\{0\} \\
d_{1}(x, y)=1}} \mu\left(\mathbb{1}_{\left\{\omega_{x} \in G_{1}, \omega_{y} \in G_{1}\right\}}\right. \\
& \left.\times\left[f\left(\Phi^{(x)}\left(\Phi^{(y)}(\omega)\right) \otimes \sigma\right)-f\left(\Phi^{(y)}(\omega) \otimes \sigma\right)\right]^{2}\right) .
\end{aligned}
$$

After the change of variable $\eta \equiv \Phi^{(y)}(\omega)$ inside the expectation, the above quantity can be bounded from above by

$$
\left.2 L \lambda_{\Phi} \sum_{\substack{x, y \in \Lambda \backslash\{0\} \\ d_{1}(x, y)=1}} \mu\left(\mathbb{1}_{\left\{\eta_{x} \in G_{1}, \eta_{y} \in G_{2}\right\}}\left[f\left(\Phi^{(x)}(\eta)\right) \otimes \sigma\right)-f(\eta \otimes \sigma)\right]^{2}\right) .
$$


Putting all together, we get that there exist a constant $c>0$ such that

$$
\begin{aligned}
\mu\left(\mathbb{1}_{0} \operatorname{Var}_{0}(f)\right) \leq & c \lambda_{\Phi} p_{2}^{-2}\left[\mu\left(\mathbb{1}_{\left\{\omega_{\vec{e}_{1}} \in G_{2}\right\}} \prod_{j=2}^{d} \mathbb{1}_{\left\{S G_{j}\right\}} \operatorname{Var}_{0}(f)\right)\right. \\
& \left.\left.+\sum_{\substack{x, y \in \Lambda \backslash\{0\} \\
d_{1}(x, y)=1}} \mu\left(\mathbb{1}_{\left\{\eta_{x} \in G_{1}, \eta_{y} \in G_{2}\right\}}\left[f\left(\Phi^{(x)}(\eta)\right) \otimes \sigma\right)-f(\eta \otimes \sigma)\right]^{2}\right)\right] .
\end{aligned}
$$

We can now analyse the first term inside the above square bracket by repeating the above analysis for the second direction. In $d-1$ steps, the proof is complete.

3.1. A canonical-paths bound on the right-hand side of (3.3). In this section, we proceed by analysing the right-hand side of (3.3) in the special case in which $S=\{0,1\}^{V}, V=\prod_{i=1}^{d}\left[n_{i}\right]$ for some integers $\left\{n_{i}\right\}_{i=1}^{d}$ and $\hat{\mu}$ is the $\operatorname{Bernoulli}(p)$ product measure. We will write $|V|$ for the cardinality of $V$. In this setting, the probability space $\left(S^{\mathbb{Z}^{d}}, \mu\right)$ becomes isomorphic to $(\Omega, \mu)$ where $\Omega=\{0,1\}^{\mathbb{Z}^{d}}$ and $\mu$ is the $\operatorname{Bernoulli}(p)$ product measure. It is therefore convenient to do a relabelling of the variables $\omega \in S^{\mathbb{Z}^{d}}$ as follows.

Let $\mathbb{Z}^{d}(\vec{n})$ be the renormalised lattice $\bigotimes_{i=1}^{d}\left(n_{i} \mathbb{Z}\right)$ and let, for $x \in \mathbb{Z}^{d}(\vec{n}), V_{x}:=$ $V+x$. We will write $x \sim y$ iff $x, y$ are nearest neighbors in the renormalised lattice $\mathbb{Z}^{d}(\vec{n})$. The old "block" variable $\omega_{x} \in S$ associated to $V_{x}$ is renamed as $\omega_{V_{x}}=$ $\left\{\omega_{y}\right\}_{y \in V_{x}}$ with now $\omega_{y} \in\{0,1\}$ for all $y$ 's. In particular, the local variance term $\operatorname{Var}_{x}(f)$ appearing in the right-hand side of (3.3) becomes $\operatorname{Var}_{V_{x}}(f)$. Accordingly, we rewrite the mapping $\Phi^{(x)}, x \in \mathbb{Z}^{d}(\vec{n})$, as $\Phi^{\left(V_{x}\right)}$.

In order to formulate our bounds, we need to define the canonical paths (cf., e.g., [28]).

DEFINITION 3.6 (canonical-paths). Let $\omega, \omega^{\prime} \in \Omega$ be two configurations which differ in finitely many vertices. We say that $\Gamma_{\omega, \omega^{\prime}} \equiv\left(\omega^{(1)}, \omega^{(2)}, \ldots, \omega^{(k)}\right)$ is a canonical-path between $\omega, \omega^{\prime}$ if (i) $\omega^{(1)}=\omega, \omega^{(k)}=\omega^{\prime}$, (ii) $\omega^{(i)} \neq \omega^{(j)}$ for all $i \neq j$ (no loops) and (iii) for any $i \in[k-1]$ the configuration $\omega^{(i+1)}$ is obtained from $\omega^{(i)}$ by a single spin flip. The integer $k$ will be referred to as the length of the path.

The bounds on the individual terms in the right-hand side of (3.3) are then as follows.

LEMMA 3.7. We assume that, for any $x \in \mathbb{Z}^{d}(\vec{n})$, any $z \in V_{x}$ and any $\omega$ such that $\omega_{V_{x}+\vec{e}_{i}} \in G_{2}$ for all $i \in[d]$, a canonical-path $\Gamma_{\omega, \omega^{z}}$ has been defined such that a generic transition in the path consists of a spin flip in $V_{x} \cup\left(\cup_{i=1}^{d}\left\{V_{x}+\vec{e}_{i}\right\}\right)$. Let

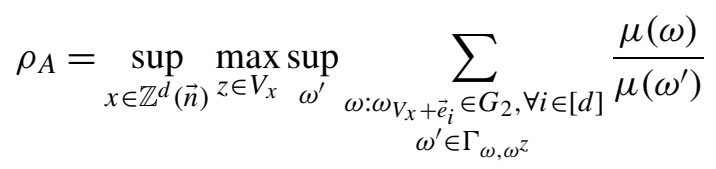


be the congestion constant of the family of canonical-paths and let $N_{A}$ be their maximal length. Then

$$
\sum_{x \in \mathbb{Z}^{d}(\vec{n})} \mu\left(\left[\prod_{i \in[d]} \mathbb{1}_{\left\{\omega_{V_{x}+\vec{e}_{i}} \in G_{2}\right\}}\right] \operatorname{Var}_{V_{x}}(f)\right) \leq c \rho_{A} N_{A}|V|^{2} \sum_{y \in \mathbb{Z}^{d}} \mu\left(\mathbb{1}_{y}^{A}(\omega) \operatorname{Var}_{y}(f)\right),
$$

for a numerical constant $c>0$, where $\mathbb{1}_{y}^{A}(\omega)$ is the indicator of the event that there exists $x \in \mathbb{Z}^{d}(\vec{n}), z \in V_{x}$ and $\bar{\omega}$ such that $\bar{\omega}_{V_{x}+\vec{e}_{i}} \in G_{2}, \forall i \in[d]$ and the pair $\left(\omega, \omega^{y}\right)$ form a transition of the canonical-path between $\bar{\omega}$ and $\bar{\omega}^{z}$.

LEMMA 3.8. We assume that, for any $x \sim y$ and any $\omega \in \Omega$ such that $\omega_{V_{x}} \in$ $G_{1}$ and $\omega_{V_{y}} \in G_{2}$, a canonical-path between $\omega$ and $\Phi^{\left(V_{x}\right)}(\omega)$ has been defined such that a generic transition in the path consists of a spin flip in $V_{x} \cup V_{y}$. Let

$$
\rho_{B}=\sup _{\omega^{\prime}} \sup _{x \sim y} \sum_{\substack{\omega: \omega_{V_{x}} \in G_{1}, \omega_{V_{y}} \in G_{2} \\ \omega^{\prime} \in \Gamma_{\omega, \Phi}\left(V_{x}\right)_{(\omega)}}} \frac{\mu(\omega)}{\mu\left(\omega^{\prime}\right)}
$$

and let $N_{B}$ be the maximal length of the paths. Then

$$
\begin{gathered}
\left.\sum_{x \sim y} \mu\left(\mathbb{1}_{\left\{\omega_{V_{x}} \in G_{1}, \omega_{V y} \in G_{2}\right\}}\left[f\left(\Phi^{\left(V_{x}\right)}(\omega)\right)-f(\omega)\right]^{2}\right)\right] \\
\leq c \rho_{B} N_{B}|V| \sum_{z \in \mathbb{Z}^{d}} \mu\left(\mathbb{1}_{z}^{B}(\omega) \operatorname{Var}_{z}(f)\right)
\end{gathered}
$$

for a numerical constant $c>0$, where $\mathbb{1}_{z}^{B}(\omega)$ is the indicator of the event that there exists $x \sim y$ and $\omega^{\prime}$ such that $\omega_{V_{x}}^{\prime} \in G_{1}, \omega_{V_{y}}^{\prime} \in G_{2}$ and the pair $\left(\omega, \omega^{z}\right)$ form a transition of the canonical-path between $\omega^{\prime}$ and $\Phi^{\left(V_{x}\right)}\left(\omega^{\prime}\right)$.

The proof of the above two lemmas is practically identical so we only prove the first one.

ProOF OF LEMMA 3.7. The starting inequality is

$$
\operatorname{Var}_{V_{x}}(f) \leq \sum_{z \in V_{x}} \mu_{V_{x}}\left(\operatorname{Var}_{z}(f)\right)
$$

For simplicity in the sequel, we assume $x=0$. Given $\omega$ such that $\omega_{V+\vec{e}_{i}} \in G_{2} \forall i \in$ $[d]$ and $z \in V$, let $\Gamma_{\omega, \omega^{z}}=\left(\omega^{(1)}, \omega^{(2)}, \ldots, \omega^{(k)}\right)$ be the corresponding canonicalpath. Then

$\operatorname{Var}_{z}(f)(\omega)=p(1-p)\left[f\left(\omega^{z}\right)-f(\omega)\right]^{2} \leq p(1-p) k \sum_{j=1}^{k}\left[f\left(\omega^{(i+1)}\right)-f\left(\omega^{(i)}\right)\right]^{2}$ 
so that

$$
\begin{aligned}
\mu\left(\mathbb{1}_{\left\{\omega_{V+\vec{e}_{i}} \in G_{2} \forall i \in[d]\right\}} \operatorname{Var}_{z}(f)\right) & \leq N_{A} p(1-p) \mu\left(\sum_{i=1}^{k-1}\left[f\left(\omega^{(i+1)}\right)-f\left(\omega^{(i)}\right)\right]^{2}\right) \\
& \leq c \rho_{A} N_{A} \sum_{y \in V \cup\left(\bigcup_{i=1}^{d} V+\vec{e}_{i}\right)} \mu\left(\mathbb{1}_{y}^{A}(\omega) \operatorname{Var}_{y}(f)\right),
\end{aligned}
$$

where $\mathbb{1}_{y}^{A}(\omega)$ is as in the statement and, after the change of variables $\omega=\omega^{(i)}$, we used the definition of $\rho_{A}$ to bound the relative density between $\omega^{(i)}$ and $\omega$. The statement of the lemma now follows at once.

For future purposes, we summarise the conclusion of our bounds.

COROLlaRY 3.9. Under the same assumptions as in Lemmas 3.7 and 3.8,

$$
\begin{aligned}
\operatorname{Var}(f) \leq & c\left(\lambda_{\Phi} p_{2}^{-4}\right)^{d}\left[\rho_{A} N_{A}|V|^{2} \sum_{z} \mu\left(\mathbb{1}_{z}^{A}(\omega) \operatorname{Var}_{z}(f)\right)\right. \\
& \left.+\rho_{B} N_{B}|V| \sum_{z} \mu\left(\mathbb{1}_{z}^{B}(\omega) \operatorname{Var}_{z}(f)\right)\right]
\end{aligned}
$$

REMARK 3.10. In the application to KCM, the choice of the canonical-paths entering in the above corollary will always be such that $\max \left(\mathbb{1}_{z}^{A}(\omega), \mathbb{1}_{z}^{B}(\omega)\right) \leq$ $c_{z}(\omega)$, where $c_{z}$ is the constraint of the KCM at $z \in \mathbb{Z}^{d}$. Thus in this case the conclusion of the Corollary implies a Poincaré inequality $\operatorname{Var}(f) \leq C \mathcal{D}(f)$, where $\mathcal{D}(f)=\sum_{z} \mu\left(c_{z} \operatorname{Var}_{z}(f)\right)$ is the Dirichlet form of the KCM (cf. Remark 2.2) and $C$ satisfies

$$
C \leq c\left(\lambda_{\Phi} p_{2}^{-4}\right)^{d}\left(\rho_{A} N_{A}|V|^{2}+\rho_{B} N_{B}|V|\right)
$$

4. Application to specific KCM models. In this section, we begin by recalling the definition of the Fredrickson-Andersen constrained spin models with $k$ facilitation (FA-kf in the sequel) introduced by H.C. Andersen and G.H. Fredrikson in [1] and of the GG-KCM. As it will be clear in a moment, the FA-kf models are closely related to the so-called $k$-neighbour model in bootstrap percolation, while the GG-KCM model is related to the anisotropic bootstrap percolation model introduced by Gravner-Griffeath [19, 20]. As such, the dynamical properties of both models near the ergodicity threshold are intimately related to the scaling properties of the corresponding bootstrap percolation models in the same regime. Finally, we state our main result relating the persistence time with the critical bootstrap percolation length. This will be proven in Section 5 using Corollary 3.9. The key step will consist in finding suitable (i.e., depending on the specific choice of the constraints) good and super-good events $G_{1}, G_{2}$, map $\phi$ and canonical-paths. 
4.1. The models. We will work with the probability space $(\Omega, \mu)$ where $\Omega=\{0,1\}^{\mathbb{Z}^{d}}$ and $\mu$ is the product $\operatorname{Bernoulli}(p)$ and we will be interested in the asymptotic regime $q \downarrow 0$ where $q=1-p$. A generic kinetically constrained model (KCM in the sequel) is a particular interacting particle system, that is, a Markov process on $\Omega$, described by the Markov generator

$$
(\mathcal{L} f)(\omega)=\sum_{x \in \mathbb{Z}^{d}} c_{x}(\omega)\left(\mu_{x}(f)-f\right)(\omega),
$$

where $\mu_{x}(f)$ is the $\operatorname{Bernoulli}(p)$-average of $f(\omega)$ w.r.t. to the variable $\omega_{x}$. The constraints $\left\{c_{x}\right\}_{x \in \mathbb{Z}^{d}}$ are defined as follows. Let $\mathcal{U}=\left\{U_{1}, \ldots, U_{m}\right\}$ be a finite collection of finite subsets of $\mathbb{Z}^{d} \backslash\{0\}$. We call $\mathcal{U}$ the update family of the process and each $X \in \mathcal{U}$ an update rule. Then $c_{x}$ is the indicator function of the event that there exists an update rule $X \in \mathcal{U}$ such that $\omega_{y}=0 \forall y \in X+x$. We emphasise that we do not assume that the constraints satisfy the exterior property of Section 2.3. Using these assumptions, it is easy to check (cf. [8] for a detailed analysis) that $\mathcal{L}$ becomes the generator of a reversible Markov process on $\Omega$, with reversible measure $\mu$.

In the FA-kf model, one takes as $\mathcal{U}$ the family of $k$-subsets of the set of nearest neighbors of the origin. In the GG-KCM model in two dimensions one takes $\mathcal{U}$ as the family of 3-subsets of the set of nearest neighbors of the origin together with the vertices $\left\{ \pm 2 \vec{e}_{1}\right\}$. In the terminology of bootstrap percolation (see, e.g., [5] and the recent survey [26]), the update family of FA-kf for $k \in[2, d]$ belongs to the class of critical balanced models and the update family of GG-KCM is critical unbalanced. Such a difference will appear clearly in the sequel.

REMARK 4.1. Given a KCM with update family $\mathcal{U}$, we will sometimes refer to the corresponding bootstrap percolation process as the monotone process defined in (1.1) using the same update rules of the KCM.

We now define the two main quantities characterising a KCM.

Definition 4.2. The relaxation time $T_{\text {rel }}(q ; \mathcal{U})$ of the generator $\mathcal{L}$ is the best constant $C$ in the Poincaré inequality

$$
\operatorname{Var}(f) \leq C \mathcal{D}(f) \quad \text { for all local } f
$$

where $\mathcal{D}(f)=\sum_{x} \mu\left(c_{x} \operatorname{Var}_{x}(f)\right)$ is the Dirichlet form associated to $\mathcal{L}$.

A finite relaxation time implies (see, e.g., [22]) that the reversible measure $\mu$ is mixing for the semigroup $P_{t}$ with exponentially decaying time auto-correlations,

$$
\operatorname{Var}\left(e^{t \mathcal{L}} f\right) \leq e^{-t / T_{\text {rel }}} \operatorname{Var}(f), \quad f \in L^{2}(\mu) .
$$


The second (random) quantity is the first time the spin at the origin reaches the zero state:

$$
\tau_{0}=\inf \left\{t \geq 0: \omega_{0}(t)=0\right\} .
$$

In the physics literature, the hitting time $\tau_{0}$ is usually referred to as the persistence time, while, in the bootstrap percolation framework, it would be more conveniently dubbed infection time. In [9], Theorem 4.7, it was proved that

$$
\mathbb{P}_{\mu}\left(\tau_{0} \geq t\right) \leq \exp \left(-q t / T_{\text {rel }}(q ; \mathcal{U})\right)
$$

implying

$$
\mathbb{E}_{\mu}\left(\tau_{0}\right) \leq T_{\text {rel }}(q ; \mathcal{U}) / q
$$

A matching lower bound in terms of $T_{\text {rel }}(q ; \mathcal{U})$ is missing and we have instead the following result whose proof is deferred to the Appendix. Recall that $\tau_{\mathrm{BP}}$ is the infection time of the origin for the corresponding bootstrap percolation process.

LEMMA 4.3. There exists $\delta=\delta(\mathcal{U}) \in(0,1)$ such that, for all $q$ small enough,

$$
\mathbb{E}_{\mu}\left(\tau_{0}\right) \geq \delta \mu\left(\tau_{\mathrm{BP}}\right) \geq \frac{\delta}{2} T_{c}(q ; \mathcal{U})
$$

One of the main results of [8] states that all the $\mathrm{KCM}$ with update family $\mathcal{U}$ such that $q_{c}(\mathcal{U})=0$ have a finite relaxation time $T_{\text {rel }}$, and thus a finite mean infection time $\mathbb{E}_{\mu}\left(\tau_{0}\right)$. In particular, the above holds for the FA-kf for $k \in[2, d]$ model and the GG-KCM and our main aim is to compute the rate at which $T_{\text {rel }}$ and $\mathbb{E}_{\mu}\left(\tau_{0}\right)$ diverge as $q \rightarrow 0$ in both cases.

4.2. Main result. We begin to recall what is known on the asymptotic scaling as $q \rightarrow 0$ of the critical length $L_{c}(q ; \mathcal{U})$ defined in (1.2) and the relaxation time $T_{\text {rel }}(q ; \mathcal{U})$, when the update family $\mathcal{U}$ is that of the FA-kf and the GG-KCM models.

For the update family $\mathcal{U}$ of the FA-kf model on $\mathbb{Z}^{d}$, it was proved in [3] (see the Introduction there for a short account of previous relevant results) that for any $d \geq k \geq 2$ there exists an explicit constant $\lambda(d, k)$ such that

$$
L_{c}(q ; k, d) \equiv L_{c}(q ; \mathcal{U})=\exp _{(k-1)}\left(\frac{\lambda(d, k)+o(1)}{q^{1 /(d-k+1)}}\right),
$$

where $\exp _{(r)}$ denotes the $r$-times iterated exponential, $\exp _{(r+1)}(x)=$ $\exp \left(\exp _{(r)}(x)\right)$. For the case of the GG-KCM, it was established in [17] (see also [16] for a detailed analysis of the $o(1)$ term below) that instead

$$
L_{c}(q ; \mathcal{U})=\exp \left(\frac{(\log (1 / q))^{2}}{12 q}(1+o(1))\right) .
$$


As far as the asymptotic behaviour $T_{\text {rel }}(q ; \mathcal{U})$ as $q \rightarrow 0$ is concerned, only the FA$\mathrm{kf}$ model has been considered so far and the following bounds have been proved in [8]. There exists $c>0$ such that

$$
\begin{aligned}
& L_{c}(q ; \mathcal{U})^{1-o(1)} \leq T_{\mathrm{rel}}(q ; \mathcal{U}) \leq \exp \left(c / q^{5}\right), \quad d=k=2, \\
& L_{c}(q ; \mathcal{U})^{1-o(1)} \leq T_{\mathrm{rel}}(q ; \mathcal{U}) \leq \exp _{(d-1)}(c / q), \quad d \geq 3, k \leq d .
\end{aligned}
$$

Notice that the above upper bounds are very far from $L_{c}(q ; \mathcal{U})$. In conclusion, while the control of the critical length $L_{c}(q ; \mathcal{U})$ is rather sharp, the relaxation time $T_{\text {rel }}(q ; \mathcal{U})$ and the mean hitting time $\mathbb{E}_{\mu}\left(\tau_{0}\right)$ are still poorly controlled. The main outcome of the theorem below is a much tighter connection between $T_{\text {rel }}(q ; \mathcal{U})$ and, therefore, $\mathbb{E}_{\mu}\left(\tau_{0}\right)$ and $L_{c}(q ; \mathcal{U})$.

THEOREM 4.4. For the FA-2f model in $\mathbb{Z}^{d}$ and the GG-KCM model, there exists $\alpha>0$ such that

$$
T_{\text {rel }}(q ; \mathcal{U})=O\left(L_{c}(q ; \mathcal{U})^{\log (1 / q)^{\alpha}}\right) .
$$

For FA-kf model in $\mathbb{Z}^{d}$ with $3 \leq k \leq d$, there exists $c>\lambda(d, k)$ such that

$$
T_{\text {rel }}(q ; \mathcal{U}) \leq \exp _{(k-1)}\left(c / q^{1 /(d-k+1)}\right) .
$$

\section{Proof of Theorem 4.4 .}

5.1. Reader's guide and notation. The proof of the theorem uses all the machinery which was developed in the previous sections. Therefore, for all the above models, the coarse-grained probability space $(S, \hat{\mu})$ (cf., e.g., the beginning of Section 3.1) will be of the form $S=\{0,1\}^{V}$, with $V=\prod_{i=1}^{d}\left[n_{i}\right]$ and $\hat{\mu}$ the product $\operatorname{Bernoulli}(p)$ measure.

The starting point of the proof is to make an appropriate choice for the value of $\vec{n}=\left(n_{1}, \ldots, n_{d}\right)$ together with a working definition of the good and super-good

events $G_{1}, G_{2} \subset S$ and of the mapping $G_{1} \stackrel{\Phi}{\mapsto} G_{2}$ (cf. Section 3 ) for each model. Clearly, in order to apply Theorem 3.2 and Corollary 3.9, our choice of ( $\left.\vec{n}, G_{1}, G_{2}\right)$ must ensure that the probabilities $p_{1}=\hat{\mu}\left(G_{1}\right)$ and $p_{2}=\hat{\mu}\left(G_{2}\right)$ satisfy the basic condition $\lim _{q \rightarrow 0}\left(1-p_{1}\right)\left(\log \left(1 / p_{2}\right)\right)^{2}=0$ of Theorem 3.2. In the FA-kf models, no direction plays a special role (it is a balanced model in the language of [26]) and, therefore, we choose $n_{i}=n$ for all $i \in[d]$. In the GG-KCM, the above symmetry is broken and we will need to distinguish between the two directions. This part of the proof is carried out in Part I (see below). The second part of the proof (cf. Part II below) involves defining appropriately the canonical-paths appearing in Lemma 3.7 and 3.8 (see also Corollary 3.9) and bounding the corresponding length and congestion constants.

Carrying out the above program could become particularly heavy from a notational point of view. Therefore, we will sometimes adopt a more descriptive and 
informal approach. More specifically, given a configuration $\omega \in\{0,1\}^{\mathbb{Z}^{d}}$ and a region $\Lambda \subset \mathbb{Z}^{d}$, we will declare $\Lambda$ empty (occupied) if $\omega \uparrow \Lambda=0(1)$. While constructing the canonical-paths appearing in Lemmas 3.7 and 3.8, we will say that we empty (fill) $\Lambda$ if we flip to 0 (1), one by one according to some preassigned schedule (i.e., an ordering of the to-do flips), all the occupied/empty sites of $\Lambda$. It is important to emphasise that the schedules involved in the operations of emptying or filling a region will always be such that each spin flip dictated by the schedule will occur while fulfilling the specific constraint of each model. Schedules with this property will be dubbed legal schedules. A closely related notion is that of legal canonical-path.

Definition 5.1. Given a KCM, let $\left\{c_{x}\right\}_{x \in \mathbb{Z}^{d}}$ be the corresponding family of constraints. A legal canonical-path between two configurations $\omega, \omega^{\prime}$ is a canonical-path $\Gamma_{\omega, \omega^{\prime}} \equiv\left(\omega^{(1)}, \omega^{(2)}, \ldots, \omega^{(m)}\right)$ with the additional property that $c_{x^{(i)}}\left(\omega^{(i)}\right)=1 \forall i \in[m-1]$, where $\omega^{x}$ denotes the configuration obtained from $\omega$ by flipping the value $\omega_{x}$ and $x^{(i)}$ is the vertex such that $\omega^{(i+1)}=\left(\omega^{(i)}\right)^{x^{(i)}}$. We say that the canonical-path is decreasing (increasing) if for any $i \in[m-1]$ and any $x \in \mathbb{Z}^{d} \omega_{x}^{(i+1)} \leq \omega_{x}^{(i)}\left(\omega_{x}^{(i+1)} \geq \omega_{x}^{(i)}\right)$.

Next, we recall the notion of a subset of $\mathbb{Z}^{d}$ being internally spanned which will play a crucial role in the definition of the good and super-good events for the specific KCM treated here.

DEFINITION 5.2 (Internally spanned). Consider a KCM with updating family $\mathcal{U}$. Given $\Lambda \subset \mathbb{Z}^{d}$, we will denote by $I(\mathcal{U}, \Lambda) \subset \Omega_{\Lambda}$ the event that $\Lambda$ is $\mathcal{U}$ internally spanned, that is,

$$
\left[\left\{x \in \Lambda: \omega_{x}=0\right\}\right]_{\mathcal{U}}=\Lambda \text {. }
$$

When the update family is that of the FA-kf model in $d$ dimensions (i.e., the update family of $k$-neighbour model), we will sometimes write $I(d, k, \Lambda)$ instead of $I(\mathcal{U}, \Lambda)$ and we will say that $\Lambda$ is $k$-internally spanned.

We will also need the following result for $k$-neighbour bootstrap percolation.

LEMMA 5.3 ([11], Lemma 4.1). There exists $\epsilon>0$ s.t. for $L \geq C L_{c}(\epsilon q ; \mathcal{U})$, $L \in \mathbb{N}$ and $C$ a large enough numerical constant,

$$
\mu\left(\omega \in I\left(d, k,[L]^{d}\right)\right) \geq 1-\exp \left(-L / L_{c}(\epsilon q ; \mathcal{U})\right) .
$$

Clearly, for any update family $\mathcal{U}$, the following holds. If $\omega$ is such that the region $\Lambda$ is $\mathcal{U}$-internally spanned and $\omega^{\prime}$ is the configuration equal to zero in $\Lambda$ and equal to $\omega$ elsewhere, then there exists a legal decreasing canonical-path $\Gamma_{\omega, \omega^{\prime}}$ 
which only uses flips inside $\Lambda$. In particular, the length of $\Gamma_{\omega, \omega^{\prime}}$ is at most $|\Lambda|$. By reversing the path, we get a legal increasing path between $\omega^{\prime}$ and $\omega$.

Before starting the actual proof, it will be useful to fix some additional notation. Given the hypercube $\Lambda=[n]^{d}$ and $i \in[d]$, we set $E_{i}(\Lambda)=\left\{x \in \Lambda: x_{j}=1, j \neq i\right\}$ and we call it the $i$ th-edge of $\Lambda$. Strictly speaking, an edge of $V$ is a set of the form $\left\{x \in V: x_{j} \in\{1, n\} \forall j \neq i\right\}$. Here, we will only need edges with one end-point at the vertex $(1, \ldots, 1)$. Any $(d-1)$-dimensional set of the form $\Lambda \cap\left\{x: x_{i}=j\right\}$, $j \in[n]$, will be called an $i$-slice and it will be denoted by $\mathrm{Sl}_{j}(\Lambda ; i)$. A generic $i$ frame $F_{j}(\Lambda ; i), j \in[n]$, is the $(d-2)$-dimensional subset of $\operatorname{Sl}_{j}(\Lambda ; i)$ consisting of the vertices $x$ such that $x_{k}=1$ for some $k \neq i$. If $\Lambda^{\prime}=x+\Lambda$, then $E_{i}\left(\Lambda^{\prime}\right)=$ $E_{i}(\Lambda)+x$, etc. If clear from the context, we will drop the specification $\Lambda$ from the notation.

5.2. Part I. Here, we define the blocks of the coarse-grained analysis together with the good and super-good events and the mapping $\Phi$. We do that separately for the FA-kf model and the GG-KCM.

5.2.1. The FA-kf model with $k \geq 3$. Let $\ell=L_{c}(\epsilon q ; k-1, d-1)$ [see (4.2)] with $\epsilon$ defined in Remark 5.3 and fix $n=A \ell \log \ell$, with $A>2(d-1)+1$.

Definition $5.4\left(G_{1}, G_{2}, \Phi\right)$. The good event $G_{1}$ consists of all $\omega \in S$ such that for all $i \in[d]$ every $i$-slice of $V$ is $(k-1)$-internally spanned. The supergood event $G_{2}$ consists of all $\omega \in G_{1}$ such that the first slice in any direction is empty. The mapping $G_{1} \stackrel{\Phi}{\mapsto} G_{2}$ is defined by $\Phi(\omega)_{x}=0$ if $x \in \bigcup_{i=1}^{d} \operatorname{Sl}_{1}(V ; i)$ and $\Phi(\omega)_{x}=\omega_{x}$ otherwise.

With the triple $\left(G_{1}, G_{2}, \Phi\right)$ we get immediately that

$$
\begin{aligned}
\left(1-p_{1}\right) & \leq d n\left(1-\hat{\mu}\left(I\left(d-1, k-1,[n]^{d-1}\right)\right)\right), \\
p_{2} & =\hat{\mu}\left(G_{2}\right) \geq p_{1} q^{d n^{d-1}}, \\
\lambda_{\Phi} & \leq\left(\frac{2}{q}\right)^{d n^{d-1}} .
\end{aligned}
$$

Using (5.1) together with the definition of $n$, we get immediately that $1-p_{1} \leq$ $A \ell^{-(A-1)} \log \ell$ so that $\lim _{q \rightarrow 0}\left(1-p_{1}\right)\left(\log \left(1 / p_{2}\right)\right)^{2}=0$ for all $A>2 d-1$.

5.2.2. The FA-kf model with $k=2$. In this case, we choose $V=\prod_{i \in[d]}\left[n_{i}\right]$ with $n_{i}=\left(\frac{A}{q} \log (1 / q)\right)^{1 /(d-1)}$ with $A>3 /(d-1)$.

Definition $5.5\left(G_{1}, G_{2}, \Phi\right)$. The good event $G_{1}$ consists of all $\omega \in S$ such that, for all $i \in[d]$ every $i$-slice of $V$ contains at least one empty vertex. The supergood event $G_{2}$ consists of all $\omega \in G_{1}$ such that any $i$-edge of $V$ is empty. The mapping $G_{1} \stackrel{\Phi}{\mapsto} G_{2}$ is defined by $\Phi(\omega)_{x}=0$ if $x \in \bigcup_{j=1}^{d} E_{j}$ and $\Phi(\omega)_{x}=\omega_{x}$ otherwise. 
As before, we easily get

$$
\begin{aligned}
1-p_{1} & =\hat{\mu}\left(G_{1}^{c}\right) \leq d n(1-q)^{n^{d-1}} \leq d n q^{A}, \\
p_{2} & =\hat{\mu}\left(G_{2}\right) \geq q^{n d}, \quad \lambda_{\Phi} \leq \frac{2^{n d}}{q^{n d}},
\end{aligned}
$$

where $2^{\text {nd }}$ is the number of possible configurations $\omega^{\prime} \in\{0,1\} \bigcup_{i} E_{i}$. In particular, for all $A>3 /(d-1), \lim _{q \rightarrow 0}\left(1-p_{1}\right)\left(\log \left(1 / p_{2}\right)\right)^{2}=0$.

5.2.3. The GG-KCM model. Here, we choose $n_{1}=\left\lfloor\frac{A \log (1 / q)}{q^{2}}\right\rfloor$ and $n_{2}=$ $\left\lfloor\frac{A \log (1 / q)}{q}\right\rfloor, A>6$.

DEFINITION 5.6. We say that $\omega \in G_{1}$ if all columns of $V=\left[n_{1}\right] \times\left[n_{2}\right]$ contain at least one empty vertex and all rows contain at least one pair of adjacent empty vertices $\left(x, x^{\prime}\right)$. We say that $\omega \in G_{2}$ if $\omega \in G_{1}$ and the first two adjacent columns of $V$ are empty. The mapping $\Phi$ is the one which empties the first two columns of $V$.

Again we easily obtain that

$$
\begin{aligned}
1-p_{1} & =O\left(q^{(A-2) / 2} \log (1 / q)\right), \\
p_{2} & =O\left(\exp \left[-\frac{2 A}{q} \log (1 / q)^{2}\right]\right), \quad \lambda_{\Phi}=O\left(2^{2 n_{2}} / q^{2 n_{2}}\right)
\end{aligned}
$$

so that $\lim _{q \rightarrow 0}\left(1-p_{1}\right)\left(\log \left(1 / p_{2}\right)\right)^{2}=0$ for $A>6$.

Notice that for all models the factor $\left(\lambda_{\Phi} / p_{2}^{4}\right)^{d}|V|$ appearing in Corollary 3.9 is bounded from above by the right-hand side of (4.3) and (4.4).

5.3. Part II. Here, we complete the proof of Theorem 4.4 by defining the canonical-paths appearing in Lemmas 3.7 and 3.8 in such a way that:

(a) they are legal canonical-paths;

(b) the congestion constants $\rho_{A}, \rho_{B}$ and the maximum length of the paths $N_{A}$, $N_{B}$ are such that $\max \left(\rho_{A} N_{a}, \rho_{B} N_{B}\right)$ is bounded from above by right-hand side of (4.3) for the FA-2f and the GG-KCM models and by the right-hand side of (4.4) for the FA-kf model, $k \geq 3$.

A very useful strategy to carry out this program is based on the following simple result.

LEMMA 5.7. Fix $\omega$ and let $\Lambda_{1}, \Lambda_{2}, \ldots, \Lambda_{N}$ be $N$ regions with the property that, for any $j$ and $k=j \pm 1$, if we empty $\Lambda_{j}$ then we can also empty $\Lambda_{k}$ by means of a legal schedule using only flips in $\Lambda_{k}$. Assume that $\omega$ is such that $\Lambda_{1}$ is empty 
and let $\omega^{\prime}$ be obtained from $\omega$ by emptying $\Lambda_{N}$. Then there exists a legal canonicalpath $\Gamma_{\omega, \omega^{\prime}}=\left(\omega^{(1)}, \ldots, \omega^{(m)}\right), m \leq 2 \sum_{i}\left|\Lambda_{i}\right|$, such that for any $j \in[m]$ the following holds. If the configuration $\omega^{(j+1)}$ is obtained from $\omega^{(j)}$ by flipping a vertex in $\Lambda_{k_{j}}$, then all the discrepancies (i.e., the vertices where they differ) between $\omega$ and $\omega^{(j)}$ are contained in $\Lambda_{k_{j}-1} \cup \Lambda_{k_{j}} \cup \Lambda_{k_{j}+1}$ if $k_{j}<N$ and in $\Lambda_{N-1} \cup \Lambda_{N}$ if $k_{j}=N$.

ProOF. By assumption, we can first empty $\Lambda_{2}$ and then $\Lambda_{3}$ by using flips first in $\Lambda_{2}$ and then in $\Lambda_{3}$. Let $\eta$ be the new configuration and let $\sigma$ be the configuration obtained from $\omega$ by emptying $\Lambda_{3}$. We can then restore the original values of $\omega$ in $\Lambda_{2}$ by reversing the legal canonical-path $\Gamma_{\sigma, \eta}$. Starting from $\sigma$, we can iteratively repeat the above procedure and get a final legal canonical-path $\Gamma_{\omega, \omega^{\prime}}$ with the required property.

REMARK 5.8. The fact that the discrepancies between an intermediate step of the path $\omega^{(j)}$ and the starting configuration $\omega$ are contained in a triple of consecutive $\Lambda_{i}$ 's allows us to easily upper bound the congestion constant $\rho_{\Gamma}:=$ $\sup _{\tilde{\omega}} \sum_{\omega: \Gamma_{\omega, \omega^{\prime}} \ni \tilde{\omega} \frac{\mu(\omega)}{\mu(\tilde{\omega})}}$ of the family $\left\{\Gamma_{\omega, \omega^{\prime}}\right\}_{\omega \in S}$ by $(2 / q)^{\max _{i}\left(\left|\Lambda_{i-2}\right|+\left|\Lambda_{i-1}\right|+\left|\Lambda_{i}\right|\right)}$. This observation will be the main tool to bound the congestion constants $\rho_{A}, \rho_{B}$ appearing in Corollary 3.9.

5.3.1. The FA-kf model with $k \geq 3$. As before, set $V=[n]^{d}$ with $n$ as in Section 5.2.1. The proof is based on a series of simple observations which, under certain natural assumptions, ensure the existence of legal canonical-paths with some prescribed properties.

CLAIM 5.9. Let $\omega$ be a configuration such that the $i$-slice $\operatorname{Sl}_{j}(V ; i)$ is empty and the $i$-slice $\mathrm{Sl}_{j-1}(V ; i)$ is $(k-1)$-internally spanned. Let $\omega^{\prime}$ be such that $\omega^{\prime} \uparrow \mathrm{Sl}_{j-1}(V ; i)=0$ and $\omega^{\prime}$ coincides with $\omega$ elsewhere. Then there is a legal decreasing canonical-path $\Gamma_{\omega, \omega^{\prime}}$ which uses only flips inside $S_{j-1}(V ; i)$; similarly, if we replace $S_{j-1}(V ; i)$ with $S_{j+1}(V ; i)$.

PROOF. The result can be immediately proven by noticing that each site in $\mathrm{Sl}_{j-1}(V ; i)$ has an empty neighbour in $\mathrm{Sl}_{j}(V ; i)$. Since $\mathrm{Sl}_{j-1}(V ; i)$ is $(k-1)$ internally spanned, the legal [w.r.t. to the FA- $(\mathrm{k}-1) \mathrm{f}$ constraint] monotone path which empties it is also legal w.r.t. the FA-kf constraint.

Claim 5.10. Fix $i \in[d], m \in[n]$ and let $\left(\omega, \omega^{\prime}\right)$ be a pair of configurations satisfying at least one of the following conditions:

(a) $\omega$ is such that the first $i$-slice is empty and all the others are $(k-1)$ internally spanned and $\omega^{\prime}$ is obtained from $\omega$ by emptying the mth $i$-slice and the first $m-1$-frames. 
(b) $\omega$ is such that $\bigcup_{i=1}^{d} \operatorname{Sl}_{1}(V ; i)$ is empty and $\omega^{\prime}$ is obtained from $\omega$ by emptying $\mathrm{Sl}_{m}(V ; i)$.

Then there exists a legal canonical-path $\Gamma_{\omega, \omega^{\prime}}=\left(\omega^{(1)}, \omega^{(2)}, \ldots, \omega^{(N)}\right)$ with $N \leq$ $2 n^{d}$ such that the only discrepancies between $\omega$ and $\omega^{(j)}, j \in[N]$, belong to the set

$$
\mathrm{Sl}_{k_{j}-1}(V, i) \cup \mathrm{Sl}_{k_{j}}(V, i) \cup \mathrm{Sl}_{k_{j}+1}(V ; i) \cup\left(\bigcup_{\ell=1}^{k_{j}} F_{\ell}(V ; i)\right) \text {, }
$$

where $k_{j}$ is such that the flip connecting $\omega^{(j)}$ to $\omega^{(j+1)}$ occurs in the $k_{j}$ th $i$-slice.

Proof. Case (a). In this case, we simply apply Lemma 5.7 and Claim 5.9 to the first $m i$-slices with a twist. After emptying the $j$ th $i$-slice, $j=1,2, \ldots, m$, instead of reconstructing the original values of $\omega$ in the previous slice we do so only in $\mathrm{Sl}_{j-1}(V ; i) \backslash F_{j-1}(V ; i)$. In such a way, the $i$-frames once emptied remain so and we get to the final configuration $\omega^{\prime}$ by a legal canonical-path satisfying the required property.

Case (b). We use again Lemma 5.7 and Claim 5.9. The base case $k=2, d=2$ follows by observing that the $i$-slices, $i=1,2$, are 1 -internally spanned since they all contain an empty site. The case $k=2$ and $d>2$ follows by induction. In fact, $\mathrm{Sl}_{2}(V ; i)$ is of the form $\Lambda \times\left\{x_{i}=2\right\}$ with $\Lambda$ isomorphic to $[n]^{d-1}$. Moreover, $\bigcup_{i=1}^{d-1} \operatorname{Sl}_{1}(\Lambda ; j) \times\left\{x_{i}=2\right\} \subset \bigcup_{j=1}^{d} \mathrm{Sl}_{1}(V ; j)$ and, therefore, it is empty by assumption. By the inductive hypothesis for $k=2, d-1$, we can empty $\operatorname{Sl}_{2}(V ; i)$ using only flips inside $\mathrm{Sl}_{2}(V ; i)$. This concludes the proof for $k=2$ and any $d \geq 2$. We thus assume the result true for $(k-1, d-1)$ and prove it for $(k, d), d \geq k$. In this case, we apply Lemma 5.7 to the regions $\Lambda_{j}:=\mathrm{Sl}_{j}(V ; i) \cup\left(\bigcup_{i=1}^{d} \mathrm{Sl}_{1}(V ; i)\right)$. For simplicity and w.l.o.g., we only verify the assumption of the lemma for the pair $\Lambda_{1}, \Lambda_{2}$. In this case, we aim at constructing a legal canonical-path that empties $\mathrm{Sl}_{2}(V ; i)$ using only flips there.

Thus, using the inductive hypothesis and the fact that each site on $\mathrm{Sl}_{2}(V ; i)$ has an additional empty neighbour in $\operatorname{Sl}_{1}(V ; i)$, we can empty $\operatorname{Sl}_{2}(V ; i)$ by a legal canonical-path which uses flips only in $\mathrm{Sl}_{2}(V ; i)$.

We are now ready to state the main result for the case under consideration.

PROPOSITION 5.11. In the above setting, there exists a choice of the canonical-paths occurring in Lemmas 3.7 and 3.8 such that, for a suitable positive constant $c$ :

- each path is a legal canonical-path and $\max \left(N_{A}, N_{B}\right) \leq c n^{d}$;

- $\max \left(\rho_{A}, \rho_{B}\right) \leq(1 / q)^{c n^{d-1}}$. 
Using that $n=A \ell \log \ell, \ell$ being the critical length for the FA-(k-1)f model in $\mathbb{Z}^{d-1}$ given by [cf. (4.2)]

$$
\ell=\exp _{(k-2)}\left(\frac{\lambda(d-1, k-1)+o(1)}{q^{1 /(d-k+1)}}\right),
$$

the proposition implies that

$$
\max \left(\rho_{A} N_{A}, \rho_{B} N_{B}\right) \leq \text { r.h.s. of (4.4), }
$$

so that the conclusion of Theorem 4.4 for the case $k \geq 3$ follows from Corollary 3.9.

PROOF OF THE PROPOSITION. We begin by examining the choice of the canonical-paths appearing in Lemma 3.8. Using the definition of the good and super-good events $G_{1}, G_{2}$ given in Section 5.2.1, our choice for the canonical paths is the one dictated by (a) of Claim 5.10. In this case, using Remark 5.8, $N_{B} \leq c n^{d}$ and $\rho_{B} \leq(1 / q)^{n^{d-1}}$ for some constant $c>0$.

We now turn to the canonical-paths appearing in Lemma 3.7. Fix $\omega$ and $z$ as in the lemma and observe that, using (b) of Claim 5.10, we can empty all the slices $S_{z_{i}+1}(V ; i), i \in[d]$, via a legal schedule. Call $\omega^{\prime}$ the configuration obtained in this way. In $\omega^{\prime}$, we can make a flip at $z$ since $z$ has at least $d$ empty neighbors. We can finally reverse the path from $\omega$ to $\omega^{\prime}$ to obtain our final legal canonical-path between $\omega$ and $\omega^{z}$. Claim 5.10 again implies that $N_{A} \rho_{A} \leq c n^{2 d} 1 / q^{c n^{d-1}}$.

5.3.2. The FA-kf model with $k=2$. As before, set $V=[n]^{d}$ with $n$ as in Section 5.2.2. For any $x \in V$, we define the cross at $x$ as the $\operatorname{set} \mathcal{C}_{x}(V):=$ $\bigcup_{i=1}^{d} \mathcal{C}_{x}(V ; i)$ with

$$
\mathcal{C}_{x}(V ; i):=\left\{x^{\prime} \in V: x_{j}^{\prime}=x_{j} \forall j \neq i\right\} .
$$

Notice that the cross of the vertex $(1,1, \ldots, 1) \in V$ is the union of the edges $E_{i}(V)$.

CLAIM 5.12. Given $x, y \in V$ such that $y=x \pm \vec{e}_{i}$ for some $i \in[d]$, let $\omega$ be such that $\mathcal{C}_{x}(V)$ is empty and let $\omega^{\prime}$ be the configuration obtained from $\omega$ by emptying the cross at $y$. Then there exists a legal decreasing canonical-path $\Gamma_{\omega, \omega^{\prime}}=\left(\omega^{(1)}, \ldots, \omega^{(m)}\right), m \leq 2 d n$, using only flips in $\mathcal{C}_{x}(V) \cup \mathcal{C}_{y}(V)$.

ProOf. Since $y=x+ \pm \vec{e}_{i}$ then necessarily $\mathcal{C}_{y}(V ; i)=\mathcal{C}_{x}(V ; i)$. Consider now the vertex $z=y \pm \vec{e}_{j}$ with $j \neq i$. This vertex has two empty neighbours: one is $y$ and another belongs to $\mathcal{C}_{x}(V)$. Therefore, $z$ can be emptied. We can iterate until we empty the $j$ th arm of the cross $\mathcal{C}_{y}(V)$ and then repeat the procedure for all the remaining direction but the $i$ th one.

As for the case $k \geq 3$, we have the following. 
PROPOSITION 5.13. In the above setting, there exists a choice of the canonical-paths occurring in Lemmas 3.7 and 3.8 such that, for a suitable positive constant $c$ :

- each path is a legal canonical-path and $\max \left(N_{A}, N_{B}\right) \leq c n^{2}$;

- $\max \left(\rho_{A}, \rho_{B}\right) \leq(1 / q)^{c n}$.

Using that $n=\left(\frac{A}{q} \log (1 / q)\right)^{1 /(d-1)}$, the proposition implies that

$$
\max \left(\rho_{A} N_{A}, \rho_{B} N_{B}\right) \leq \text { r.h.s. of (4.3), }
$$

so that the conclusion of Theorem 4.4 for the case $k=2$ follows from Corollary 3.9 .

ProOF OF PROPOSITION 5.13. We begin by examining the choice of the canonical-paths appearing in Lemma 3.8. Fix $\omega$ and suppose that we have two hypercubes $V=[n]^{d}$ and $V^{\prime}=V+(n+1) \vec{e}_{1}$ such that $\omega \uparrow V$ is good and $\omega \uparrow V^{\prime}$ is super-good. Let also $\omega^{\prime}$ be obtained from $\omega$ by emptying the cross of the vertex $(1,1, \ldots, 1) \in V$ so that $\omega^{\prime} \uparrow V$ is super-good. Let now $z^{(i)}$ be the first (according to some a priori order) vertex in the $(n-i+1)$ th 1 -slice $\operatorname{Sl}_{n-i+1}(V ; 1)$, which is empty and let $\bar{z}^{(i)}=z^{(i)}+\vec{e}_{1}$. Observe that the vertex $\bar{z}^{(i)}$ belong to the same 1 -slice of $V$ as the vertex $z^{(i-1)}$ and that the vertex $z^{(i)}$ exists for all $i \in[n]$ because $\omega \uparrow V$ is good. Finally, let $\gamma=\left(x^{(1)}, \ldots x^{(m)}\right), m \leq n^{2}$, be the geometric path connecting $x^{(1)}:=(1, \ldots, 1)+n \vec{e}_{1} \in V^{\prime}$ with $x^{(m)}:=(1, \ldots, 1) \in V$ constructed according to the following schedule:

(a) join $x^{(1)}$ with $\bar{z}^{(1)}$ by first adjusting the second coordinate, then the third one, etc.;

(b) join $\bar{z}^{(1)}$ to $z^{(1)}$;

(c) repeat the above steps with $x^{(1)}$ replaced by $z^{(1)}$ and $\bar{z}^{(1)}$ by $\bar{z}^{(2)}$, etc.

Next, for $i \in[m]$, let $\Lambda_{i}$ be the cross $\mathcal{C}_{x^{(i)}}\left(V^{(i)}\right)$ where $V^{(i)}$ is the hypercube $V+\left(x_{1}^{(i)}-1\right) \vec{e}_{1}$. Notice that $x^{(i)} \in \mathrm{Sl}_{1}\left(V^{(i)} ; 1\right)$. We claim that the above sets satisfy the assumption of Lemma 5.7. If the hypercubes $V^{(i)}, V^{(i+1)}$ are the same, then the claim follows immediately from Claim 5.12. If $V^{(i+1)}=V^{(i)}-\vec{e}_{1}$, then necessarily the pair $\left(x^{(i)}, x^{(i+1)}\right)$ must be of the form $\left(\bar{z}^{(j)}, z^{(j)}\right)$ for some $j$ and having the cross $\mathcal{C}_{x^{(i)}}\left(V^{(i)}\right)$ empty implies that also the cross $\mathcal{C}_{x^{(i)}}\left(V^{(i+1)}\right)$ is empty because, by assumption, $\omega_{z^{(j)}}=0$. Thus we can apply again Claim 5.12, this time in the hypercube $V^{(i+1)}$ and empty $\Lambda_{i+1}$. It is now a simple check to verify that the path defined in this way satisfy $N_{B} \leq c n^{2}$ and $\rho_{B} \leq e^{c n}$ for some constant $c>0$.

We now examine the canonical-paths entering in Lemma 3.7. Let $\omega$ be such that all the hypercubes $V+\vec{e}_{i}, i \in[d]$, are super-good, let $z \in V$ and let $\omega^{\prime}$ be obtained from $\omega$ by flipping $\omega_{z}$; w.l.o.g. we assume in the sequel that $z=(1, \ldots, 1)$.

Let $\tilde{\omega}$ be the intermediate configuration obtained from $\omega$ by emptying the cross (in $V$ ) of the vertex $x^{(1)}:=(n, \ldots, n)$. Using Lemma 5.12, it is easy to 
check that there exists a legal canonical-path $\Gamma_{\omega, \tilde{\omega}}$ with a congestion constant $\rho_{\Gamma} \leq(1 / q)^{c n}$ for some constant $c>0$. Next, let $\gamma=\left(x^{(1)}, \ldots, x^{(m)}\right)$ be a geometric path connecting $x^{(1)}$ with the vertex $z+\sum_{i=1}^{d} \vec{e}_{i}$ and define $\Lambda_{i}=\mathcal{C}_{x^{(i)}}(V)$. Using Claim 5.12 and the definition of $\tilde{\omega}$, the sets $\left\{\Lambda_{i}\right\}_{i=1}^{m}$ satisfy the assumption of Lemma 5.7. In conclusion, we have proved the existence of a legal canonicalpath $\Gamma_{\omega, \hat{\omega}}$ where $\hat{\omega}$ is obtained from $\omega$ by emptying the cross of $x^{(m)}$. Now we can legally flip $z$ and then reverse the path $\Gamma_{\omega, \hat{\omega}}$ to finally get to $\omega^{\prime}=\omega^{z}$. In conclusion, we have obtained a legal canonical-path $\Gamma_{\omega, \omega^{\prime}}$ and the claimed properties of $N_{A}$ and $\rho_{A}$ follow at once from its explicit construction.

5.3.3. The GG-KCM model. Recall that in this case the basic block $V$ is the $\left[n_{1}\right] \times\left[n_{2}\right]$ rectangle, with $n_{1}, n_{2}$ as in Section 5.2.3. Moreover, given $\omega \in\{0,1\}^{V}$, the block $V$ is good if every column contains an empty site and every row contains a pair of adjacent empty sites. It is super-good if it is good and the first two columns are empty.

In this setting, two basic observations will be at the basis of our definition of the canonical-paths appearing in Lemmas 3.7 and 3.8. Fix an integer $n$ together with $\omega \in\{0,1\}^{[4] \times[n+1]}$ and consider four consecutive columns $C_{i}=\{x=(i, j), j \in$ $[n]\}, i \in[4]:$

(1) If $C_{1}, C_{2}$ are empty and $C_{3}$ contains an empty site, then $C_{3}$ can be emptied by a legal decreasing canonical-path using only flips in $C_{3}$; similarly, if the role of $C_{1}$ and $C_{3}$ is interchanged.

(2) If $C_{1}, C_{2}$ are empty and the two vertices $x=(3, n+1)$ and $y=(4, n+1)$ above, the 3 rd and 4 th column are also empty, then $C_{3}$ and $C_{4}$ can be emptied by a legal decreasing canonical-path using only flips in $C_{3} \cup C_{4}$. Similarly, if the role of the pair $\left(C_{1}, C_{2}\right)$ and $\left(C_{3}, C_{4}\right)$ is interchanged and the sites $x, y$ are replaced by $x^{\prime}=(1, n+1), y^{\prime}=(2, n+1)$.

Using the above, we can prove our final proposition.

Proposition 5.14. For the GG-KCM model, there exists a choice of the canonical-paths occurring in Lemmas 3.7 and 3.8 such that, for a suitable positive constant $c$ :

- each path is a legal canonical-path and $\max \left(N_{A}, N_{B}\right) \leq c n_{1} n_{2}$;

- $\max \left(\rho_{A}, \rho_{B}\right) \leq(1 / q)^{c n_{2}}$.

PROOF. We begin with the definition of the canonical-paths appearing in Lemma 3.8 with, for simplicity, $V_{x}=V$ and $V_{y}=V^{\prime}$ where $V^{\prime}$ is either $V+$ $\left(n_{1}+1\right) \vec{e}_{1}$ or $V+\left(n_{2}+1\right) \vec{e}_{2}$. For simplicity, we will not make any attempt to optimize our construction, that is, to improve over the constant $c$ above.

In the first case, $V^{\prime}=V+\left(n_{1}+1\right) \vec{e}_{1}$, let $\omega \in\{0,1\}^{V \cup V^{\prime}}$ be such that $V$ is good and $V^{\prime}$ is super-good and let $\omega^{\prime}$ be obtained from $\omega$ by emptying the first two 


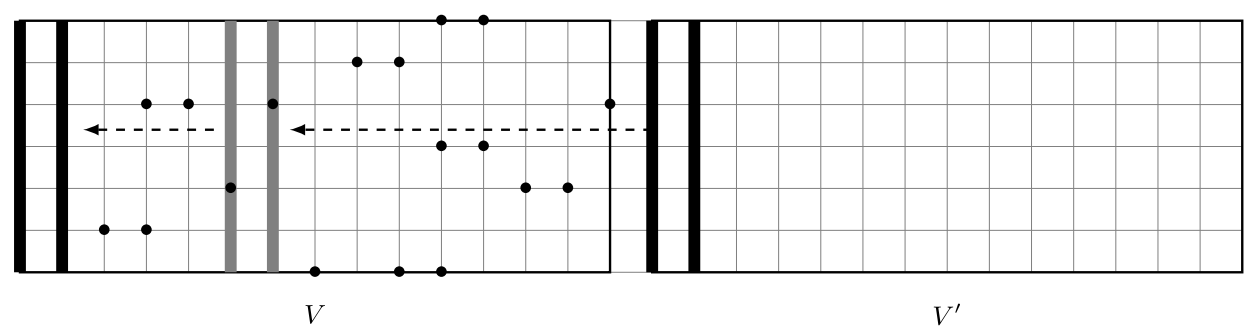

FIG. 3. A sketch of the canonical-path $\Gamma_{\omega, \omega^{\prime}}$ appearing in Lemma 3.8 for two horizontally adjacent blocks. Only the 1 st and 2 nd empty columns of the right super-good block are drawn (black). The black dots in the left block denote the empty sites, while the gray columns denote the different positions of the pair of adjacent columns inside the path. Notice the pair of adjacent empty sites on each row.

columns of $V$. Then we can use observation (1) above together with Lemma 5.7 to get that there exists a legal canonical-path $\Gamma_{\omega, \omega^{\prime}}$ of maximal length $c n_{1} n_{2}$ and congestion constant $\rho_{B} \leq(1 / q)^{c n_{2}}$ for some constant $c>0$. Notice that in this case we did not use the fact that if $V$ is good then every row contains a pair of adjacent empty sites (cf. Figure 3).

In the second case, $V^{\prime}=V+\left(n_{2}+1\right) \vec{e}_{2}$, for $i \in[n]$ define $a_{i}$ as the smallest integer $j \in[n-1]$ such that $x=(j, n-i+1)$ and $y=(j+1, n-i+1)$ are both empty. Using that $V$ is good, the integer $a_{i}$ is well-defined. Let also $\Lambda_{i}$ denotes the two semi-columns in $V \cup V^{\prime}$ above the vertices $\left(a_{i}, n-i+1\right)$ and $\left(a_{i}+1, n-i+1\right)$ (cf. Figure 4).

Using observation (1) together with Lemma 5.7, we can then obtain a legal canonical-path between $\omega$ and $\omega^{\prime}$, whose length is at most $c n_{1} n_{2}^{2}$ and whose congestion constant is bounded from above by $(1 / q)^{c n_{2}}$ for some $c>0$ independent of $i$, as follows:

(a) starting from the first two empty columns in $V^{\prime}$, we begin to empty $\Lambda_{1}$. Then, starting from the two empty semi-columns $\Lambda_{1} \cup\left\{a_{1}, n\right\} \cup\left\{a_{1}+1, n\right\}$, we empty the two sites $x=(1, n), x^{\prime}=(2, n)$ while restoring the original values of $\omega$ in all the other sites of $V \cup V^{\prime}$.

(b) We now repeat the same procedure with $\Lambda_{1}$ replaced by $\Lambda_{2}$ and $\left(x, x^{\prime}\right)$ replaced by $\hat{x}=(1, n-1), \hat{x}^{\prime}=(2, n-1)$, starting from the two empty semicolumns obtained by adding to the first two columns of $V^{\prime}$ the empty sites $(1, n)$, $(2, n)$.

(c) We iterate until reaching $\omega^{\prime}$.

It remains to consider the construction of the canonical-paths appearing in Lemma 3.7 and for that we use both (1) and (2) above.

Fix $\omega$ such that $V_{1}:=V+\left(n_{1}+1\right) \vec{e}_{1}$ and $V_{2}:=V+\left(n_{2}+1\right) \vec{e}_{2}$ are super-good, let $z \in V$ and let $\omega^{\prime}=\omega^{z}$. For simplicity and w.l.o.g., we assume $z=(1,1)$. We can then obtain a legal canonical-path between $\omega$ and $\omega^{\prime}$ with the required properties as follows (see Figure 5): 


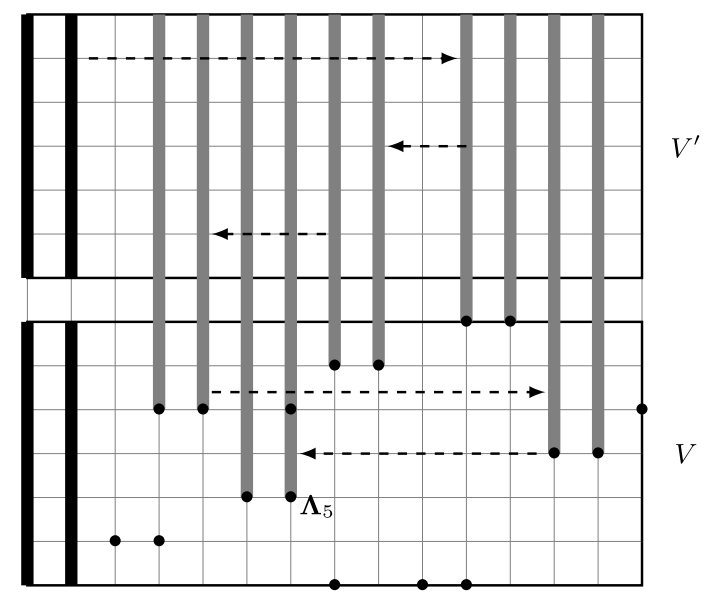

FIG. 4. A sketch of the canonical-path $\Gamma_{\omega, \omega^{\prime}}$ for two vertically adjacent blocks. The sequence of the dashed arrows must be read from top to bottom. Initially, the 1 st and 2nd empty columns of the top block (drawn in thick black) travel until they sit above the first pair of adjacent empty sites on the top row of the bottom block (grey position). At this time, their height grows by one unit. Later in the path, this new pair of empty columns is moved above the first pair of adjacent empty sites on the next to top row of the bottom block and so forth until the 1 st and 2 nd columns of the bottom block become empty.

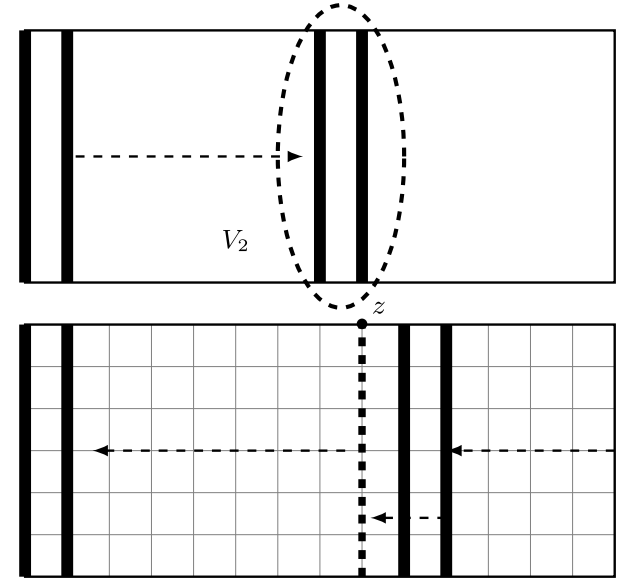

V

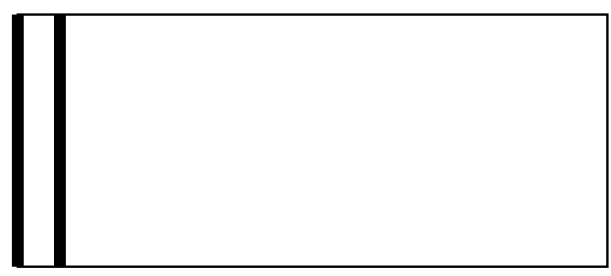

$V_{1}$

FIG. 5. A sketch of the canonical-path $\Gamma_{\omega, \omega^{\prime}}$ appearing in Lemma 3.7. Assuming that the path has been able to empty the two black columns of $V$, then it is possible to move these two columns one step further to the left as follows. First, move the initial pair of double empty columns in $V_{2}$ to the new position encircled by the dashed ellipse then, starting with the vertex $z$, empty the dashed black column in $V$ and finally restore the original values of $\omega$ to the right of $x$ and then in $V_{2}$. 
(a) by combining observation (1) with Lemma 5.7 we first empty the last two columns of $V_{2}$ without doing any flip inside $V \cup V_{1}$;

(b) at this stage, the last two columns of $V_{2}$ are empty because of (a) and the first two columns of $V_{1}$ are also empty because $V_{1}$ was super-good. Thus, using observation (2), we empty the last two columns of $V$;

(c) finally, we restore the original configuration in $V_{2}$ by reverting the path in the first step.

(d) We repeat the above three steps with a twist: we first empty the 4th and 3rd last column of $V_{2}$, then the 4th and 3rd last column of $V$. We then restore the original configuration $\omega$ in the last two columns of $V$ and, subsequently, we finally restore $\omega$ in $V_{2}$. We have now reached the intermediate configuration obtained from $\omega$ by emptying the 4 th and 3rd last column of $V$.

(e) We iterate the above step until reaching the configuration obtained from $\omega$ by emptying the 2 nd and 3rd column of $V$.

(f) Finally, using again (2) above and Lemma 5.7, we empty the vertex $(1,2)$. At this stage, we can do a flip in the corner $(1,1)$ since the vertices $(1,2),(2,1)$ and $(3,1)$ are all empty.

(g) The final step is to retrace the steps of the path which emptied $(1,2)$ and then those of the path which emptied the 2nd and 3rd column of $V$ in such a way that we end up in the configuration $\omega^{\prime}$.

\section{APPENDIX: PROOF OF LEMMA 4.3}

Fix $\omega \in \Omega$ and let $\tau_{\mathrm{BP}}(\omega ; x)$ denote the bootstrap infection time of a generic site $x$ when the initial set of infected sites coincides with the set of empty sites of $\omega$. Given a sequence $\left\{x_{i}\right\}_{i=1}^{n}$ of vertices of $\mathbb{Z}^{d}$, we set $\omega^{(0)}=\omega$ and for any $i \in[n]$ we denote by $\omega^{(i)}$ the configuration obtained from $\omega$ by setting equal to 0 all the variables at $x_{1}, \ldots, x_{i}$. We then say that $\left\{x_{i}\right\}_{i=1}^{n}$ is a sequence of legal infections if, for all $i \in[n]$ :

(i) $\omega_{x_{i}}=1$;

(ii) there exists $U_{i} \in \mathcal{U}$ s.t. $\omega_{y}^{(i-1)}=0$ for all $y \in x_{i}+U_{i}$.

Notice that, necessarily, $\tau_{\mathrm{BP}}\left(\omega, x_{j}\right) \leq j$ for $j \in[n]$.

The key reduction property of a sequence of legal infections is that it is possible to extract a subsequence $\left\{x_{i_{k}}\right\}_{k=1}^{m}$ of length $m=\tau_{\mathrm{BP}}\left(\omega, x_{n}\right)$ such that $i_{m}=n$ and

$$
\left|x_{i_{j}}-x_{i_{j+1}}\right| \leq r \quad \forall j \in[m-1],
$$

with $r=\max _{X \in \mathcal{U}} \max _{x \in X}|x|$. Indeed, let $t_{i}:=\tau_{\mathrm{BP}}\left(\omega, x_{i}\right)$. If $t_{n}=1$, there is nothing to be proved. If $t_{n}>1$, then necessarily there exists $j<n$ such that:

- $x_{j}$ belongs to one of the update rules of $x_{n}$, and thus $\left|x_{j}-x_{n}\right| \leq r$,

- $t_{j} \geq t_{n}-1$, 
since otherwise $\tau_{\mathrm{BP}}\left(\omega, x_{n}\right)<t_{n}$. Let $j_{*}$ be the largest one such integers and set $i_{m-1}=j_{*}$. Then, using that $\left\{x_{i}\right\}_{i=1}^{j_{*}}$ is also a sequence of legal infections, we can repeat the argument and proceed backward until identifying the claimed subsequence $\left\{x_{i_{k}}\right\}_{k=1}^{m}$.

Let now $t \equiv \tau_{\mathrm{BP}}(\omega, 0)$ and fix $\delta \in(0,1)$. In the sequel, it will be useful to think of the KCM dynamics as built according to the standard graphical construction of an interacting particle system with a Glauber dynamics (see, e.g., [8]). In this setting, suppose that for any $r$-path $\gamma$ of length $t$ ending at the origin, that is, a sequence of $t$ vertices $\left(v_{1}, \ldots, v_{n}\right)$, with $v_{t}=0$ and $\left|v_{i}-v_{i+1}\right| \leq r$ for all $i \in$ $[t-1]$, it is not possible to find a ordered sequence $t_{1}<\cdots<t_{t}$ in $(0, \delta t)$ of rings of the Poisson clocks such that the $i$ th-ring occurs at $v_{i}$. Using the reduction property of any sequence of legal infections, we conclude that, deterministically, the KCM dynamics starting from $\omega$ cannot infect the origin within time $\delta t$.

Finally, we claim that the above assumption is satisfied w.h.p. if $\delta$ is small enough. In fact, for any given $r$-path $\gamma$ ending at the origin, the probability that there exists an ordered sequence $t_{1}<\cdots<t_{t}$ in $(0, \delta t)$ as above, is just the probability that a Poisson random variable of mean $\delta t$ is larger than $t$. Since the number of such paths is bounded by $e^{c(r) t}$, the claim follows immediately for $\delta$ small enough.

In conclusion, we have proved that there exists $\delta>0$ such that, for any $\omega$ such that $\tau_{\mathrm{BP}}(\omega, 0) \geq 1$,

$$
\mathbb{P}_{\omega}\left(\tau_{0} \geq \delta \tau_{\mathrm{BP}}(\omega, 0)\right) \geq 1 / 2
$$

Acknowledgements. We are deeply in debt to R. Morris for several enlightening and stimulating discussions on bootstrap percolation models. We also acknowledge the hospitality of our respective departments during several exchange visits and the organizers of the 2016 Oberwolfach's workshop "Large Scale Stochastic Dynamics" for their hospitality in a stimulating atmosphere.

\section{REFERENCES}

[1] Andersen, H. C. and Fredrickson, G. H. (1984). Kinetic Ising model of the glass transition. Phys. Rev. Lett. 53 1244-1247.

[2] Balister, P., Bollobás, B., Przykucki, M. and Smith, P. (2016). Subcritical $\mathcal{U}$ bootstrap percolation models have non-trivial phase transitions. Trans. Amer. Math. Soc. 368 7385-7411. MR3471095

[3] Balogh, J., Bollobás, B., Duminil-Copin, H. and Morris, R. (2012). The sharp threshold for bootstrap percolation in all dimensions. Trans. Amer. Math. Soc. 364 26672701. MR2888224

[4] Berthier, L. and Biroli, G. (2011). Theoretical perspective on the glass transition and amorphous materials. Rev. Modern Phys. 83 587-645.

[5] Bollobas, B., Duminil-Copin, H., Morris, R. and Smith, P. (2014). Universality of two-dimensional critical cellular automata. Available at arXiv:1406.6680. 
[6] Bollobás, B., Duminil-Copin, H., Morris, R. and SMith, P. (2017). The sharp threshold for the Duarte model. Ann. Probab. 45 4222-4272. MR3737910

[7] BollobÁs, B., Smith, P. and Uzzell, A. (2015). Monotone cellular automata in a random environment. Combin. Probab. Comput. 24 687-722. MR3350030

[8] Cancrini, N., Martinelli, F., Roberto, C. and Toninelli, C. (2008). Kinetically constrained spin models. Probab. Theory Related Fields 140 459-504. MR2365481

[9] Cancrini, N., Martinelli, F., Roberto, C. and Toninelli, C. (2009). Facilitated spin models: Recent and new results. In Methods of Contemporary Mathematical Statistical Physics. Lecture Notes in Math. 1970 307-340. Springer, Berlin. MR2581609

[10] Cancrini, N., Martinelli, F., Roberto, C. and Toninelli, C. (2015). Mixing time of a kinetically constrained spin model on trees: Power law scaling at criticality. Probab. Theory Related Fields 161 247-266. MR3304752

[11] Cerf, R. and MAnzo, F. (2002). The threshold regime of finite volume bootstrap percolation. Stochastic Process. Appl. 101 69-82. MR1921442

[12] Chayes, J. T. and Chayes, L. (1986). Percolation and random media. In Phénomènes Critiques, Systèmes Aléatoires, Théories de Jauge, Part I, II (Les Houches, 1984) 1001-1142. North-Holland, Amsterdam. MR0880545

[13] Chleboun, P., Faggionato, A. and Martinelli, F. (2014). Time scale separation and dynamic heterogeneity in the low temperature East model. Comm. Math. Phys. 328 955993. MR3201217

[14] Chleboun, P., Faggionato, A. and Martinelli, F. (2016). Relaxation to equilibrium of generalized East processes on $\mathbb{Z}^{d}$ : Renormalization group analysis and energy-entropy competition. Ann. Probab. 44 1817-1863. MR3502595

[15] DuARTE, J. A. M. S. (1989). Simulation of a cellular automat with an oriented bootstrap rule. Phys. A 157 1075-1079.

[16] Duminil Copin, H., van Enter, A. and Hulshof, T. (2016). Higher order corrections for anisotropic bootstrap percolation. Available at arXiv:1611.03294v1.

[17] Duminil-Copin, H. and VAn EnTer, A. C. D. (2013). Sharp metastability threshold for an anisotropic bootstrap percolation model. Ann. Probab. 41 1218-1242. MR3098677

[18] Garrahan, J. P., Sollich, P. and Toninelli, C. (2011). Kinetically constrained models. In Dynamical Heterogeneities in Glasses, Colloids, and Granular Media (L. Berthier, G. Biroli, J.-P. Bouchaud, L. Cipelletti and W. van Saarloos, eds.) Oxford Univ. Press. Preprint. Available at arXiv:1009.6113.

[19] Gravner, J. and GriffeAth, D. (1996). First passage times for threshold growth dynamics on $\mathbf{Z}^{2}$. Ann. Probab. 24 1752-1778. MR1415228

[20] GRAVNER, J. and GRIFFEATH, D. (1999). Scaling laws for a class of critical cellular automaton growth rules. In Random Walks (Budapest, 1998). Bolyai Soc. Math. Stud. 9 167-186. János Bolyai Math. Soc., Budapest. MR1752894

[21] GrimmetT, G. (1999). Percolation, 2nd ed. Grundlehren der Mathematischen Wissenschaften [Fundamental Principles of Mathematical Sciences] 321. Springer, Berlin. MR1707339

[22] LiggetT, T. M. (1985). Interacting Particle Systems. Grundlehren der Mathematischen Wissenschaften [Fundamental Principles of Mathematical Sciences] 276. Springer, New York. MR0776231

[23] Marêché, L., Martinelli, F. and Toninelli, C. (2018). Energy barriers and the infection time for the kinetically constrained Duarte model. In preparation.

[24] Martinelli, F., Morris, R. and Toninelli, C. (2018). On the persistence time of kinetically constrained models: Universality in two dimensions. In preparation.

[25] Martinelli, F. and Toninelli, C. (2013). Kinetically constrained spin models on trees. Ann. Appl. Probab. 23 1967-1987. MR3134727

[26] Morris, R. (2017). Bootstrap percolation, and other automata. European J. Combin. 66250 263. MR3692148 
[27] Mountford, T. S. (1995). Critical length for semi-oriented bootstrap percolation. Stochastic Process. Appl. 56 185-205. MR1325219

[28] SALOFf-Coste, L. (1997). Lectures on finite Markov chains. In Lectures on Probability Theory and Statistics (Saint-Flour, 1996). Lecture Notes in Math. 1665 301-413. Springer, Berlin. MR1490046

Dipartimento di Matematica E Fisica

UNIVERSITÀ ROMA TRE

LARGO S.L. MURIALDO 00146

ROMA

ITALY

E-MAIL: martin@mat.uniroma3.it
LABORATOIRE DE PROBAbILITÉS ET MODÈLES ALÈATOIRES

CNRS-UMR 7599

UNIV. PARIS VI-VII

BAt.SOPHIE GERMAIN

CASE COURRIER 7012

75205 PARIS CEDEX 13

FRANCE

E-MAIL: cristina.toninelli@upmc.fr 